\title{
PRL2 links magnesium flux and sex- dependent circadian metabolic rhythms
}

\author{
Noriko Uetani, ${ }^{1}$ Serge Hardy, ${ }^{1}$ Simon-Pierre Gravel, ${ }^{1}$ Silke Kiessling, ${ }^{2}$ Adam Pietrobon, ${ }^{1}$ \\ Nau Nau Wong, ${ }^{1}$ Valérie Chénard, ${ }^{1}$ Nicolas Cermakian, ${ }^{2}$ Julie St-Pierre, ${ }^{1,3}$ and Michel L. Tremblay ${ }^{1,3,4}$ \\ ${ }^{1}$ Rosalind and Morris Goodman Cancer Research Centre, ${ }^{2}$ Laboratory of Molecular Chronobiology, Douglas Mental Health \\ University Institute, and ${ }^{3}$ Department of Biochemistry, ${ }^{4}$ Department of Medicine, Division of Experimental Medicine, \\ McGill University, Montréal, Québec, Canada.
}

\begin{abstract}
Magnesium $\left(\mathrm{Mg}^{2+}\right)$ plays pleiotropic roles in cellular biology, and it is essentially required for all living organisms. Although previous studies demonstrated intracellular $\mathrm{Mg}^{2+}$ levels were regulated by the complex of phosphatase of regenerating liver 2 (PRL2) and $\mathrm{Mg}^{2+}$ transporter of cyclin $\mathrm{M}$ (CNNMs), physiological functions of PRL2 in whole animals remain unclear. Interestingly, $\mathbf{M g}^{2+}$ was recently identified as a regulator of circadian rhythm-dependent metabolism; however, no mechanism was found to explain the clock-dependent $\mathrm{Mg}^{2+}$ oscillation. Herein, we report PRL2 as a missing link between sex and metabolism, as well as clock genes and daily cycles of $\mathbf{M g}^{2+}$ fluxes. Our results unveil that PRL2-null animals displayed sex-dependent alterations in body composition, and expression of PRLs and CNNMs were sex- and circadian time-dependently regulated in brown adipose tissues. Consistently, PRL2-KO mice showed sex-dependent alterations in thermogenesis and in circadian energy metabolism. These physiological changes were associated with an increased rate of uncoupled respiration with lower intracellular $\mathrm{Mg}^{2+}$ in PRL2-KO cells. Moreover, PRL2 deficiency causes inhibition of the ATP citrate lyase axis, which is involved in fatty acid synthesis. Overall, our findings support that sex- and circadian-dependent PRL2 expression alter intracellular $\mathrm{Mg}^{2+}$ levels, which accordingly controls energy metabolism status.
\end{abstract}

Conflict of interest: The authors have declared that no conflict of interest exists.

Submitted: November 14, 2016

Accepted: May 23, 2017

Published: July 6, 2017

Reference information:

JCI Insight. 2017;2(13):e91722. https:// doi.org/10.1172/jici.insight.91722

\section{Introduction}

Magnesium $\left(\mathrm{Mg}^{2+}\right)$ is an essential intracellular cation that regulates a broad range of cellular functions and various signaling pathways (1). Since $\mathrm{Mg}^{2+}$ is a required cofactor for virtually all ATP/GTP-dependent (adenosine/guanosine triphosphate-dependent) enzymatic reactions, physiological processes including energy metabolism are greatly affected by the intracellular $\mathrm{Mg}^{2+}$ concentration $(2,3)$. Thus, the activity of about 600 enzymes is $\mathrm{Mg}^{2+}$ dependent, but little is known about the regulation of $\mathrm{Mg}^{2+}$ transport leading to critical function of $\mathrm{Mg}^{2+}$ for proper cellular homeostasis (1). Recently, Feeney et al. reported a novel physiological function for $\mathrm{Mg}^{2+}$ in cellular circadian regulation. Namely, they observed that rhythmic changes of intracellular $\mathrm{Mg}^{2+}$ concentration regulated cellular timekeeping and energy balance (4). This finding shed light on the importance of rhythmic $\mathrm{Mg}^{2+}$ levels on circadian regulation of cellular energy metabolism (5). However, the regulatory mechanisms generating such intracellular $\mathrm{Mg}^{2+}$ rhythms are still unclear.

The 3 members in the PRL (phosphatase of regenerating liver) subfamily (PRL1, -2, and -3, also known as PTP4A1, -2, and-3) have initially received great attention as potential therapeutic targets against metastatic tumors (reviewed in refs. 6, 7). Although oncogenic properties of the PRLs leave no doubt, neither biological substrates nor physiological functions have been clearly demonstrated until recently. Notably, previous studies revealed that PRL1, -2 , and -3 control intracellular $\mathrm{Mg}^{2+}$ levels by interacting with the $\mathrm{Mg}^{2+}$ transporter of the cyclin $\mathrm{M}(\mathrm{CNNM})$ family $(8,9)$, and the interaction between PRL2 and CNNM3 is required to promote breast tumor growth through a $\mathrm{Mg}^{2+}$-dependent mechanism (10). These findings paved the way to the understanding of PRL functions in a normal physiological context, especially in $\mathrm{Mg}^{2+}$-dependent cellular processes.

PRL proteins possess a highly conserved protein tyrosine phosphatase (PTP) domain and are classified as a dual-specificity subgroup of phosphatases (DSPs) (11). PRLs are small proteins $(\sim 20 \mathrm{kDa})$ that include a carboxyl terminal CAAX motif, which allows for localization to the plasma membrane upon prenylation (12). Amino acid identities among different species and between the 3 PRL homologs 
Table 1. Genotypes of pups from heterozygous mating

\begin{tabular}{ccccc|cccc}
\hline & \multicolumn{3}{c|}{ O Day (at birth) } & \multicolumn{5}{c}{ 4 weeks } \\
& WT & Het & KO & $(n=)$ & WT & Het & KO & $(n=)$ \\
\hline $129 \times$ C57BL/6 & & & & & & & & \\
Male & $25 \%$ & $49 \%$ & $26 \%$ & $(69)$ & $26 \%$ & $55 \%$ & $18 \%$ & $(163)$ \\
\hline Female & $30 \%$ & $38 \%$ & $31 \%$ & $(86)$ & $23 \%$ & $58 \%$ & $18 \%$ & $(137)$ \\
C57BL/6 & & & & & & & & \\
\hline Male & $26 \%$ & $51 \%$ & $23 \%$ & $(53)$ & $22 \%$ & $65 \%$ & $12 \%$ & $(89)$ \\
\hline Female & $32 \%$ & $52 \%$ & $17 \%$ & $(60)$ & $33 \%$ & $63 \%$ & $4 \%$ & $(57)$ \\
\hline Expected (\%) & $25 \%$ & $50 \%$ & $25 \%$ & & $25 \%$ & $50 \%$ & $25 \%$ & \\
\hline & & & & & & & & \\
\hline
\end{tabular}

are quite high (13), yet each PRL protein exhibits distinct tissue expression levels and patterns (1417). These results suggest that, through evolution, each PRL has acquired divergent physiological functions in animals. Among the 3 PRLs, PRL 2 is the most ubiquitously expressed in mammals (15, 17). Moreover, analysis of PRL2-deficient mice revealed that PRL2 is required for proper placental development (18), spermatogenesis (19), and hematopoietic stem cell self-renewal (20).

In this study, we report that PRL2 is required for proper energy metabolism in a sex-dependent manner by controlling intracellular $\mathrm{Mg}^{2+} \mathrm{lev}$ els. Our results show circadian control of PRL2 expression in animals, and phenotypic analysis of the PRL2-KO mice establishes its involvement in daily rhythms of behavior and metabolism. We thus elucidate PRL2's role in controlling intracellular $\mathrm{Mg}^{2+}$ levels in a sex- and time-dependent manner. Taken together, our findings position PRL2 as a key regulator of the molecular mechanisms underlying cellular energy metabolism.

\section{Results}

Early-life viability of the PRL2-KO mice depends on genetic background and sex. In order to characterize the physiological functions of PRL2 in whole animals, PRL2 -KO mice were generated using the Prl2 gene-trapped ES cells (clone AQ0673) obtained from the Sanger Institute GeneTrap Resource. As shown in Supplemental Figure 1A (supplemental material available online with this article; https://doi.org/10.1172/jci. insight.91722DS1), the trapping vector was inserted between exon 1 and exon 2 of the Prl2 gene (Ptp4a2), generating PRL2 protein-null mutants (Supplemental Figure 1B). The PRL2-KO mice were maintained both in 129P2/OlaHsd:C57BL/6N $(129 \times B 6)$ mixed genetic background and in C57BL/6N (B6) strain backcrossed over 6 generations. In both genetic backgrounds, genotypes of newborn pups were similar to the expected Mendelian inheritance for heterozygous crossing (Table 1), suggesting that PRL2-KO embryos were viable during the gestation period. Although we found healthy-appearing PRL2-KO mice at 4 weeks of age, approximately $30 \%$ of male and female PRL2-KO mice of $129 \times \mathrm{B} 6$ background died during the early postnatal period (Figure 1A). In the B6 background, the survival rates of PRL2-KO mice were further decreased both in males and females compared with the mice with the $129 \times \mathrm{B} 6$ background (Table 1 and Supplemental Figure 1C). Surprisingly, female PRL2-KO mice of B6 background were the most susceptible to PRL2 deficiency, as over $80 \%$ of female PRL2-KO were found dead within 3 days after birth (Supplemental Figure 1C). Taken together, these results indicate that PRL2 deletion in mice results in an important phenotypic defect associated with sex and genetic background.

PRL2-KO mice exhibit reduced body weight and altered body composition. In both mixed 129×B6 and inbred B6 genetic backgrounds, surviving young adult PRL2-KO mice appeared generally healthy without showing any pathological signs. Consistent with a previous report that indicated growth retardation of PRL2KO mice (18), our surviving PRL2-KO mice exhibited reduced body weight throughout birth to adulthood (Figures 1, B and C, and Supplemental Figure 1, D and E). Interestingly, reduction in body weight gain was most evident during the lactation periods of PRL2-KO mice, whereas there was no difference in weight gain after 6 weeks of age when their growth had reached a steady state (Figure 1C and Supplemental Figure 1E, lower panels). Since most of the female PRL2-KO mice with B6 genetic background died at early postnatal stage, further analysis was performed on the $129 \times \mathrm{B} 6$ mixed background mice.

In order to assess their body composition, we measured tissue weight and normalized by naso-anal body length (Figure 2, A-C, and Supplemental Figure 2). Consistent with lower body weight, body length was significantly shorter in PRL2-KO mice compared with WT littermates (Figure 2A). There were no differences in brain, thymus, heart, kidney, and liver weight between PRL2-KO and WT mice (Figure 2C and Supplemental Figure 2). Spleen weight was reduced in both sexes, as reported previously (20), while the weights of both the white adipose tissue (WAT) and the brown adipose tissue (BAT) were significantly reduced in male PRL2-KOs, but not in female KOs (Figure 2, B and C). In agreement with these findings, histological analyses also revealed the size of adipocyte in WAT (Figure 2, D and E) and the percentage 
A

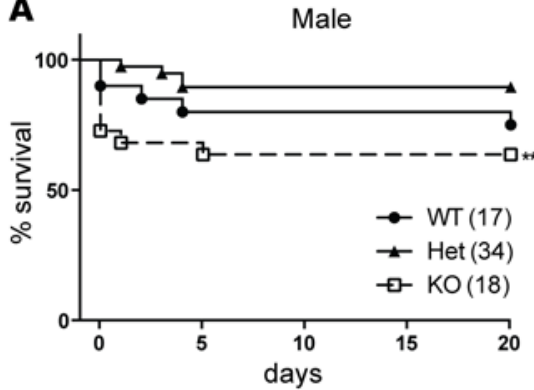

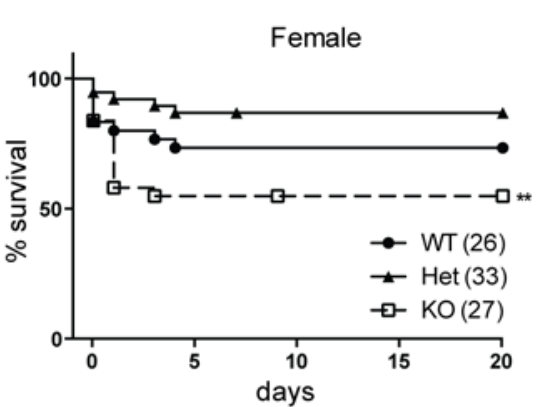

Day 0 (at birth)

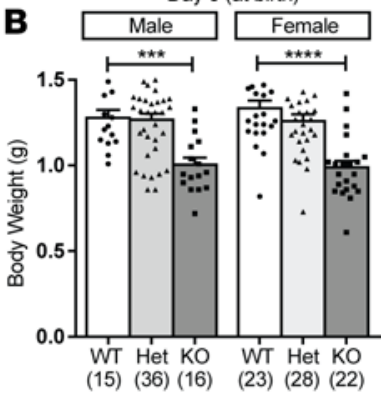

C

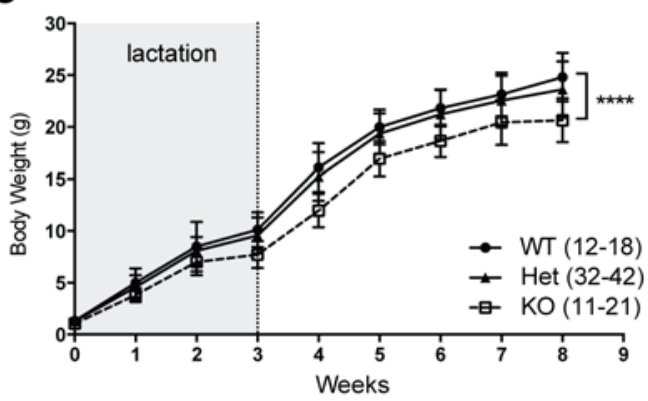

Male

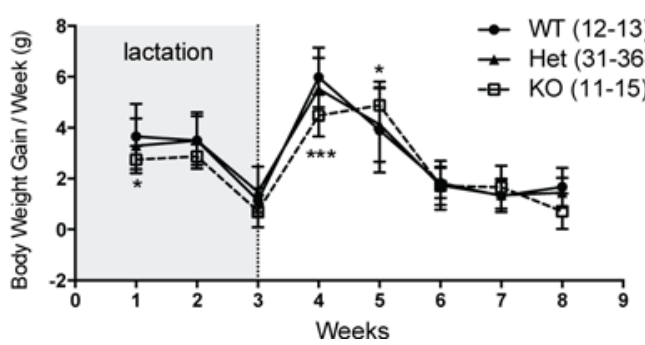

Female

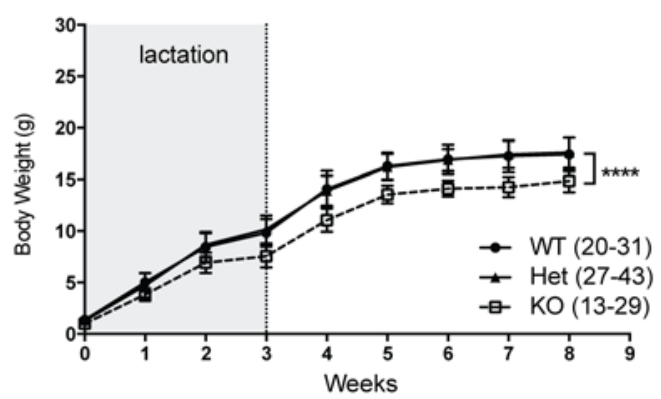

Female

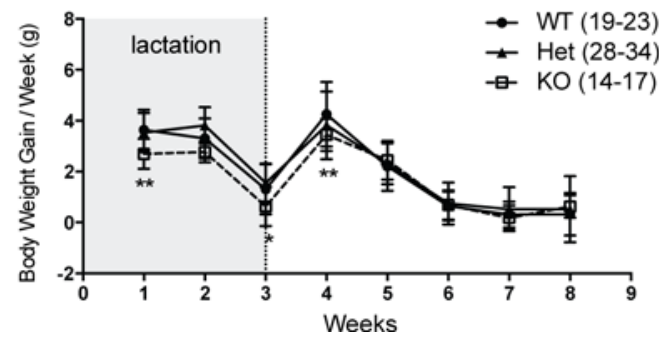

Figure 1. Viability and growth curve of the PRL2-KO mice. (A) Percent of mouse survival after birth was plotted for both male (left) and female (right). (B) Average body weight at birth for each genotype are shown. (C) Average body weight for each genotype (top) and body weight gain per week (bottom) were plotted every week from birth to 8 weeks. Data were obtained from mice in 129/B6 mixed background. Data is expressed as mean \pm SEM. Number of animals analyzed is indicated in parentheses in the figures. $P$ values were calculated by log-rank (Gehan-Breslow-Wilcoxon) test (A), one-way ANOVA with Dunnett's multiple comparison test (B and $\mathbf{C}$, bottom), and two-way ANOVA (C, top). ${ }^{*} P<0.05$; ${ }^{* *} P<0.01 ;{ }^{* *} P<0.001 ;{ }^{* * * *} P<0.0001$.

of lipid droplet area in BAT (Figure 2, D and F) were significantly reduced in male PRL2-KO vs. WT males. Lung weight was increased only in male PRL2-KO mice (Supplemental Figure 2). Moreover, muscle weight and muscle fiber size were significantly reduced only in female PRL2-KOs compared with WT littermates (Figure 2, B-D and G, and Supplemental Figure 2). These results show that PRL2 is required for proper maintenance of sex-specific body composition.

Sex-specific expression of PRL2 and CNNM Mg ${ }^{2+}$ transporters in BAT. To clarify in which tissues PRL2 could exert its physiological function, tissue distribution of PRL2 protein was examined by Western blotting using a validated PRL1/2 antibody (8) (Figures 3A and Supplemental Figure 3). Although PRL2 was ubiquitously expressed, we found different levels of PRL2 (and PRL1) expression between males and females in BAT. Higher levels were found in females (Figure 3, A and B), suggesting a sex-specific regulation of both PRLs in this tissue. Similarly, the expression levels of the $\mathrm{Mg}^{2+}$ transporter CNNM3, one of the PRL binding partners, was also elevated in female BAT compared with male BAT (Figure 3C). In addition, mRNA expression levels of the $\mathrm{Mg}^{2+}$ transporter $\mathrm{Cnnm}_{2}$ splice variants 1 and 2 (Cnnm2-1 and -2), and Cnnm3-1 were significantly upregulated in females compared with males (Figure 3D). No difference in mRNA levels of the Cnnms were detected between WT and PRL2-KO mice in both sexes. Still, intriguingly, both PRL1 and CNNM3 protein levels were increased in male KO BAT compared with sex-matched WT mice (Figure 3, B and C). Together, these results suggest that $\mathrm{Mg}^{2+}$ levels via the PRL/CNNM axis might 
A

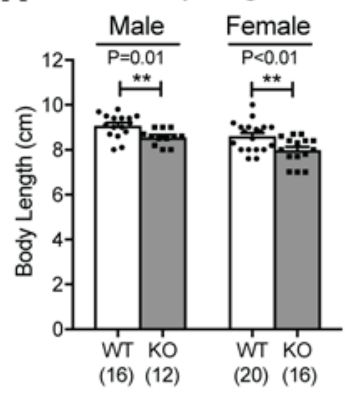

C

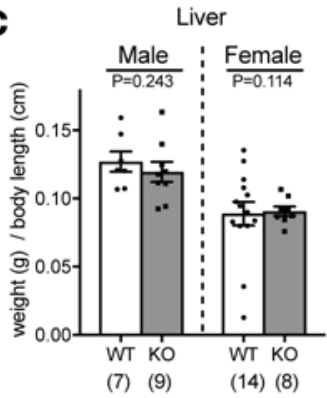

B

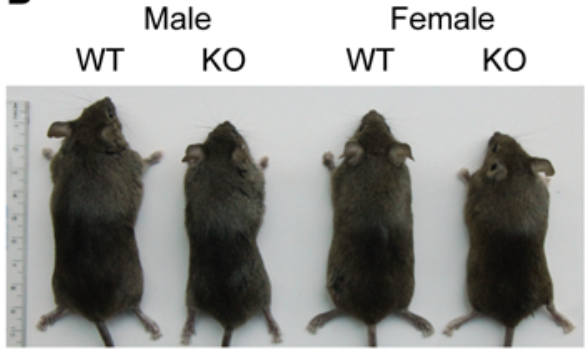

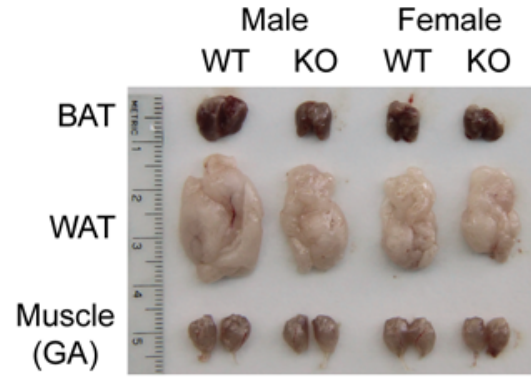

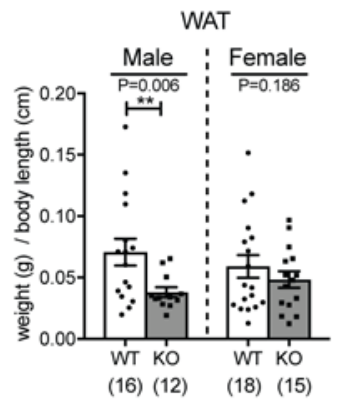

BAT

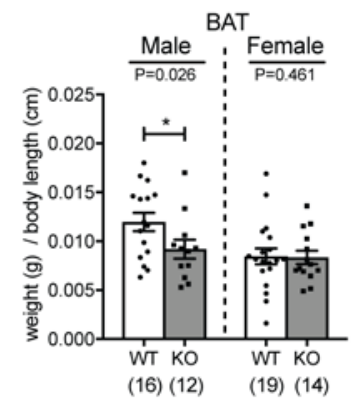

BAT

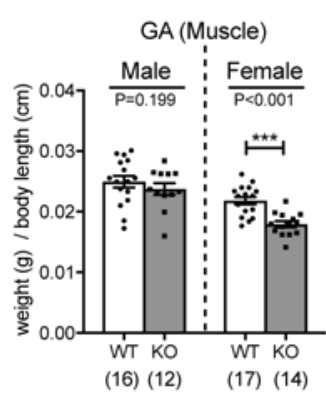

D

WAT

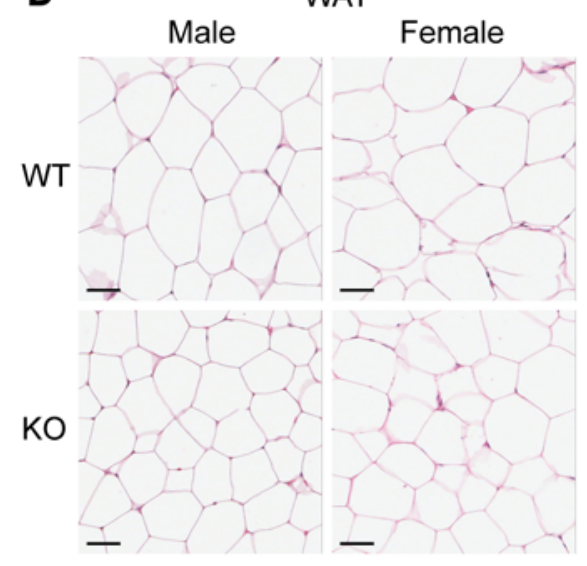

Male

Female

Muscle (GA)
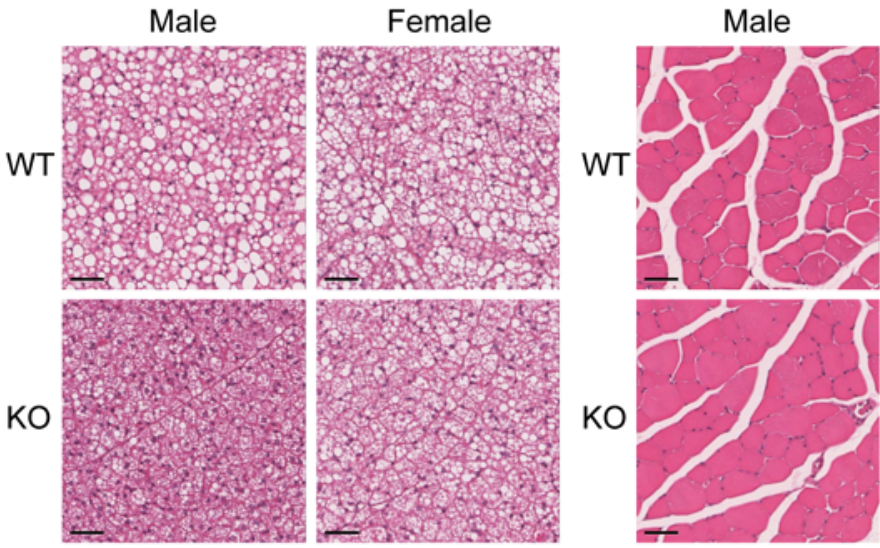

Female

E

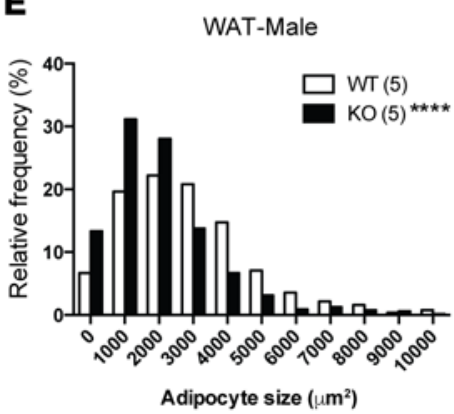

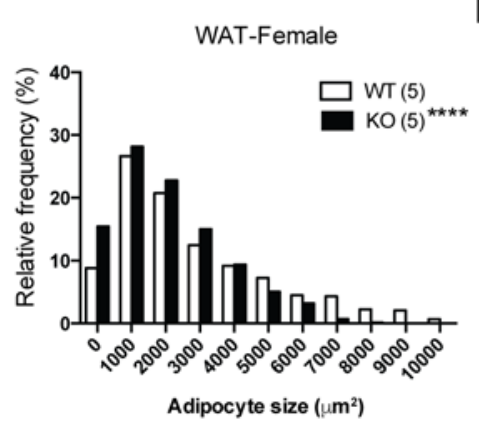
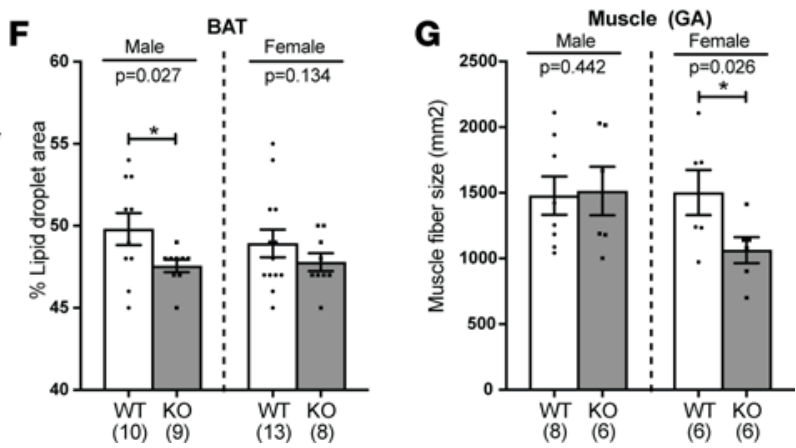

Figure 2. PRL2-KO mice exhibit altered body composition. (A) Average nose-to-anus body length is shown for each genotype. (B) Representative picture of the PRL2-deficient mice (left) and dissected gonadal white adipose tissue (WAT), brown adipose tissues (BAT), and gastrocnemius muscle (CA) are shown (right). (C) Dissected tissue weights were normalized by body length, and mean values were plotted. (D) Representative picture of H\&E-stained histological sections for gonadal WAT (left), BAT (middle), and GA muscle (right) are shown. (E) Adipocyte size (mm²) of gonadal WAT was measured, and data are expressed as histograms (percentage of total count). (F) Percent of lipid droplet area was measured on H\&E-stained histological sections of BAT obtained from WT and PRL2-KO mice. (C) Muscle fiber size $\left(\mathrm{mm}^{2}\right)$ was measured on H\&E-stained transversal histological sections of GA muscles. Data is expressed as mean \pm SEM. Number of animals analyzed is indicated in parenthesis in the figures. $P$ values were calculated by Student's $t$ test $(\mathbf{A}, \mathbf{C}, \mathbf{F}, \mathbf{G})$ and Mann-Whitney test (E). ${ }^{*} P<0.05 ;{ }^{*} P<0.01 ;{ }^{* *} P<0.001 ;{ }^{* * *} P<0.0001$. 
be differentially regulated between males and females in BAT. Also, the results suggest a compensatory mode of regulation at the translational levels to maintain proper intracellular $\mathrm{Mg}^{2+}$ balance in this tissue when PRL2 is absent.

In order to verify whether PRL2 is expressed in parenchymal cells within BAT and muscle tissues, $\mathrm{X}$-gal staining was performed on histological sections (Figure $4 \mathrm{~A}$ ) as the $\beta$-Geo ( $\beta$-galactosidase and the neomycin-resistance fusion) reporter gene was inserted in the Prl2 gene (Supplemental Figure 1A). We confirmed that $\beta$-galactosidase activity was present primarily in brown adipocytes and skeletal muscle fibers (Figure 4A), both cell types that play important roles in energy metabolism and thermogenesis. CNNM3 protein were also detected in brown adipocytes (Figure 4B). Subcellular localization of PRL2 and CNNM3 protein were further verified by coexpressing GFP-tagged PRL2 and mCherry-tagged CNNM3 in HeLa cells. As shown in Figure 4C, both PRL2 and CNNM3 proteins were detected in the cytoplasm and plasma membrane. Most importantly, they were highly colocalized on plasma membrane.

PRL2-KO mice display altered thermogenesis and susceptibility to $\mathrm{Mg}^{2+}$ deficiency. The phenotype observed in BAT and muscle of PRL2-KO mice (Figure 2) prompted us to characterize thermogenesis in these mice. When resting body temperature was measured under a housing temperature of $23^{\circ} \mathrm{C}$, female PRL2-KO mice presented a higher body temperature than WT females, while there was no difference in males (Figure 5A). We next exposed mice to $4^{\circ} \mathrm{C}$ and monitored their core body temperature. The acute cold response was impaired in PRL2-KO in both sexes (Figure 5B), suggesting that muscle-shivering thermogenesis was diminished in PRL2-KO mice.

We previously demonstrated that PRL2 was required for cellular $\mathrm{Mg}^{2+}$ homeostasis (8). Since changes in $\mathrm{Mg}^{2+}$ levels affect cellular energy metabolism (1), the metabolic phenotypes of PRL2-KO mice might stem from $\mathrm{Mg}^{2+}$ alterations. To evaluate impact of dietary $\mathrm{Mg}^{2+}$ deficiency on body weight and body temperature, mice were fed $\mathrm{Mg}^{2+}$-control diet $\left(0.05 \% \mathrm{Mg}^{2+}\right.$ by weight) or $\mathrm{Mg}^{2+}$-deficient diet $\left(<0.003 \% \mathrm{Mg}^{2+}\right.$ by weight) for a week (schematic experimental design is shown in Supplemental Figure 4). Systemic $\mathrm{Mg}^{2+}$ deficiency had a great impact on body weight, as WT mice lost weight after a week on $\mathrm{Mg}^{2+}$-deficient diet (Figure 5C). Noteworthy, female PRL2-KO mice lost body weight even with $\mathrm{Mg}^{2+}$-control diets to an extent similar to WT mice fed with the $\mathrm{Mg}^{2+}$-deficient diet. Since female PRL2-KO mice gained a comparable amount of body weight with WT with $\mathrm{Mg}^{2+}$-rich regular diets $(0.2 \% \mathrm{Mg}$ by weight) (Figure $1 \mathrm{C})$, differences of dietary $\mathrm{Mg}^{2+}$ content would be responsible for weight loss observed in female PRL2-KO mice even with $\mathrm{Mg}^{2+}$-control diets. In contrast to female PRL2-KO mice, male PRL2-KO mice were able to maintain their body weight while on the $\mathrm{Mg}^{2+}$-control diet, indicating that female PRL2-KO mice required higher amount of dietary $\mathrm{Mg}^{2+}$ than males to maintain their body weight.

Male PRL2-KO mice did not show differences in body temperature with $\mathrm{Mg}^{2+}$-rich regular diets (Figure 5A). Interestingly, both female PRL2-KO mice and male PRL2-KO mice showed higher body temperatures compared with WT mice (WT vs. KO; female, $P<0.01$; male, $P<0.0001$; Figure 5D). Additionally, we detected a significant increase in body temperature associated with $\mathrm{Mg}^{2+}$ deficiency in female WT mice (Figure 5D). Taken together, our results demonstrate that systemic $\mathrm{Mg}^{2+}$ deficiency causes weight loss and alters body temperature, similar to absence of PRL2, and KO of PRL2 lowers the threshold of $\mathrm{Mg}^{2+}$ deficiency.

Decreased intracellular $\mathrm{Mg}^{2+}$ levels associate with increased uncoupled respiration in PRL2-KO cells. $\mathrm{Mg}^{2+}$ is an important determinant of mitochondrial functions and affects bioenergetic pathways in the cell (21). Consistent with our previous data, which shows knockdown of PRL2 to decrease $\mathrm{Mg}^{2+}$ transport (8), intracellular $\mathrm{Mg}^{2+}$ levels were reduced in PRL2-KO mouse embryonic fibroblasts (MEFs) compared with cells rescued with PRL2 WT (Figure 6A). To determine the physiological relevance of this reduction in intracellular $\mathrm{Mg}^{2+}$ content, we quantified the activity of mitochondrial pathways by measuring cellular respiration. PRL2-KO cells displayed an increased rate of uncoupled respiration (Figure 6B). This suggests that their mitochondrial metabolism is less coupled to ATP production. In contrast to PRL2 WT, PRL2 C/S mutant, which lacks the ability to bind CNNMs (8), affected neither intracellular $\mathrm{Mg}^{2+}$ levels nor mitochondrial respiration when it was expressed in PRL2-KO MEFs (Figure 6, A and B). These results suggest that PRL2 exerts its functions by forming a complex with CNNMs to regulate cellular $\mathrm{Mg}^{2+}$ homeostasis and mitochondrial respiration.

In PRL2-KO BAT, we found that AMPK, which senses the ATP:AMP ratio and is activated when this ratio is low, was more phosphorylated/activated (Figure $6 \mathrm{C}$ ). Although differences were minor, systemic $\mathrm{Mg}^{2+}$ deficiency upregulated AMPK phosphorylation in WT BAT, causing a greater impact in PRL2-KO 
A

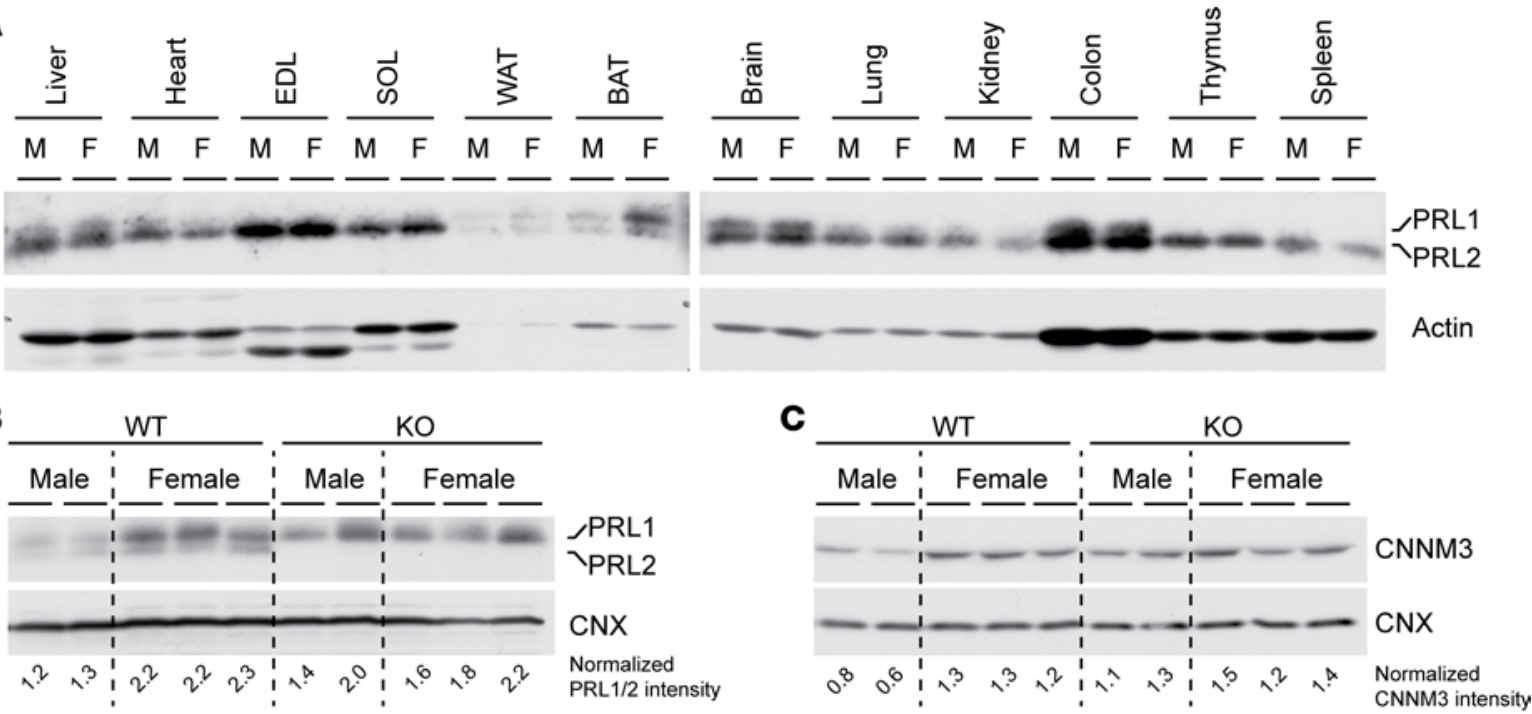

D

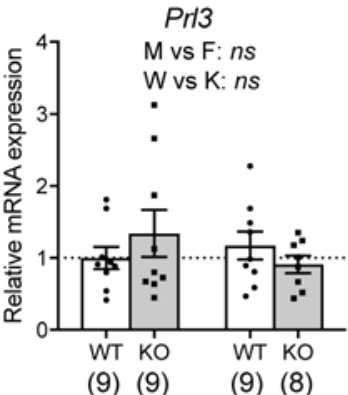

Male

Cnnm3-1

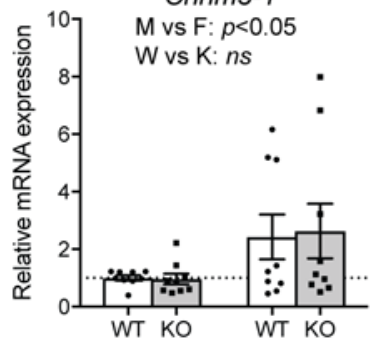

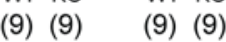

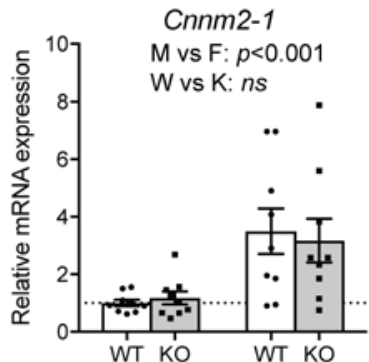

(9) (9)

Male

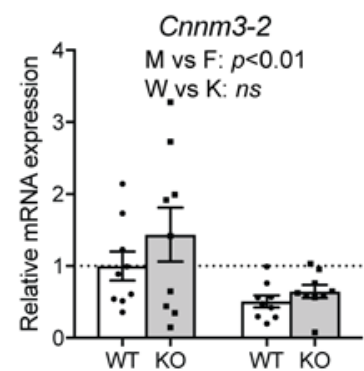

(9) (9)
(9) (9)

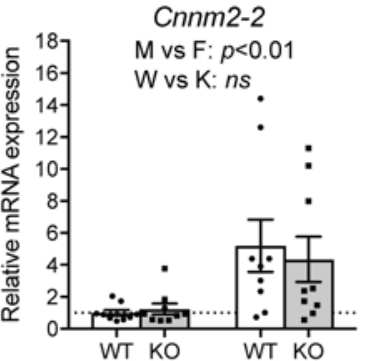

$\begin{array}{ll}\text { (9) }(9) & \text { (9) (9) }\end{array}$

Male Female

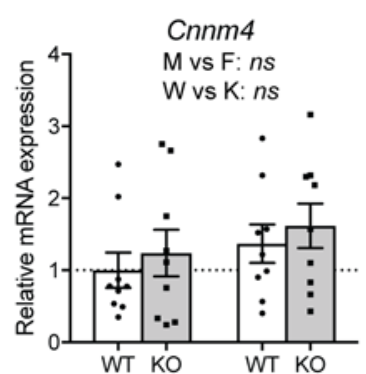

$\begin{array}{ll}\text { (9) }(9) & \text { (9) (9) }\end{array}$

Figure 3. Sex-dependent PRL1/2 and CNNM expression in BAT. (A) Tissue distribution of PRL1 and PRL2 proteins is shown. Tissues were lysed and immunoblotted with PRL1/2 and $\beta$-actin antibodies. EDL, extensor digitorum longus muscle; SOL, soleus muscle. (B and C) Protein expression of PRL1/2 (B) and CNNM3 (C) in BAT is shown. Tissue lysates were immunoblotted with PRL1/2, CNNM3, and calnexin (CNX) antibodies. Band intensities were normalized to those of CNX and indicated below the blots. (D) Quantitative PCR analyses were performed on RNA extracted from BAT. Data were normalized to Actb and expressed as fold change vs. WT. Data is expressed as mean \pm SEM. Number of animals analyzed is indicated in parentheses in the figures. $P$ values were calculated by two-way ANOVA. M vs. F, male versus female; W vs. K: WT versus KO. $P>0.05$.

BAT (Figure 6C). In addition, expression levels of the mitochondrial uncoupler uncoupling protein-1 (UCP1) were increased in BAT of PRL2-KOs (Figure 6D). These results are in agreement with the shift toward more uncoupled respiration and less coupled respiration seen in KO MEFs. UCP1-mediated nonshivering BAT thermogenesis is required for proper maintenance of body temperature and body weight (22). Accordingly, alterations in mitochondrial respiration could consequently cause higher body temperatures, cause reduced body weight, and impact body composition as we observed in PRL2-KO mice. As systemic $\mathrm{Mg}^{2+}$ deficiency induced PRL1/2 and CNNM3 protein expression in WT males or females (Figure 6, E-G), this suggests that PRL1/2 and CNNM3 are required for physiological adaptation to $\mathrm{Mg}^{2+}$ deficiency in BAT. However, we note this to be different in males, implying that the threshold of $\mathrm{Mg}^{2+}$ required is altered. 
A

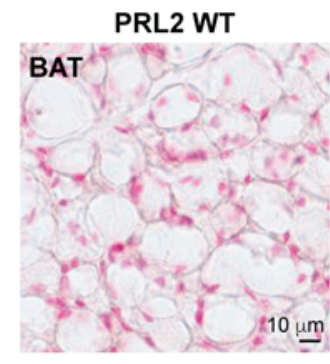

B

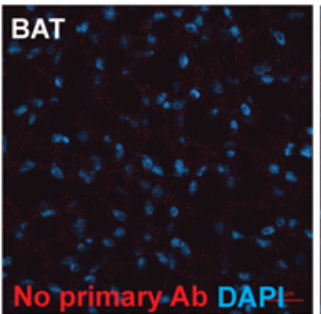

C
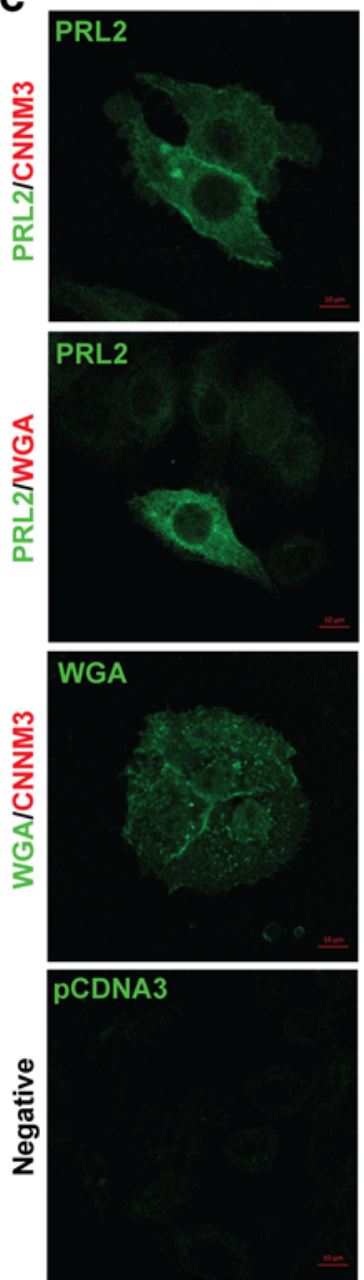

PRL2 Het
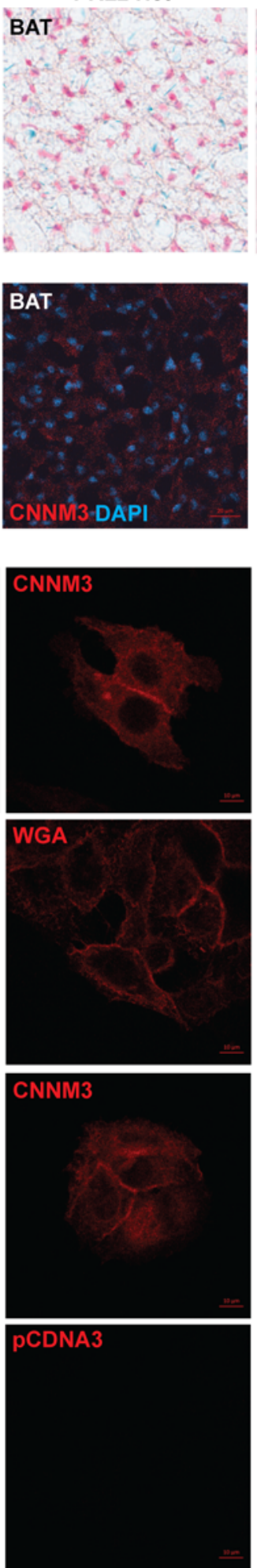

PRL2 WT

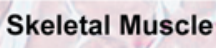

$10 \mu \mathrm{m}$

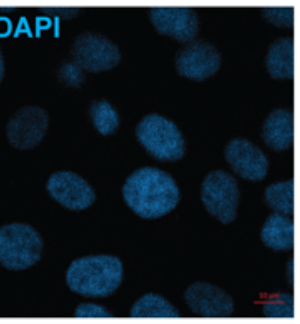

\section{DAPI}

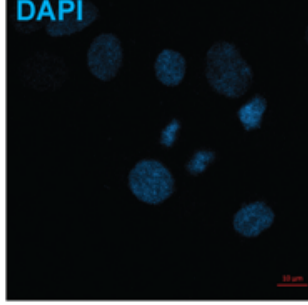

\section{DAPI}
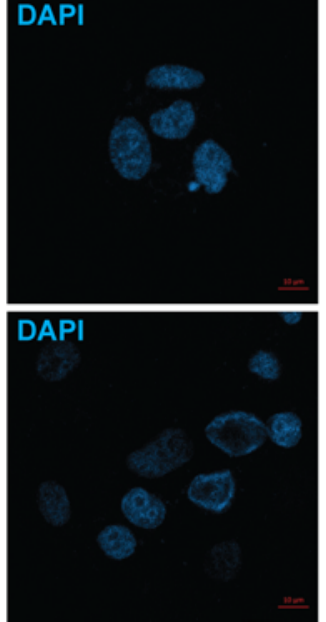

PRL2 Het
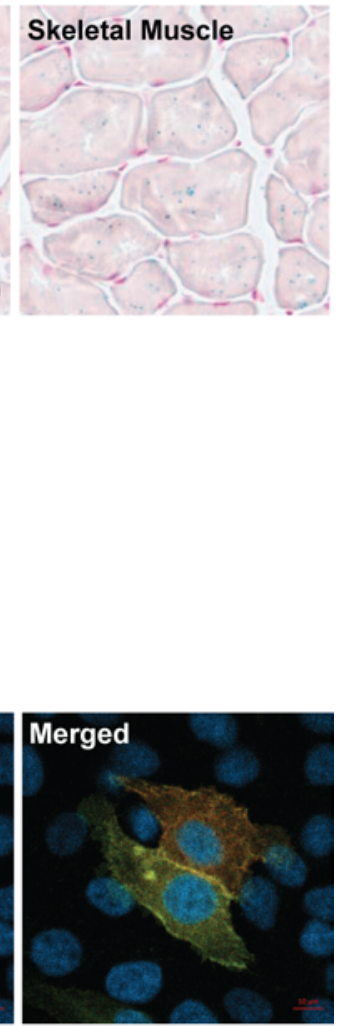

Merged

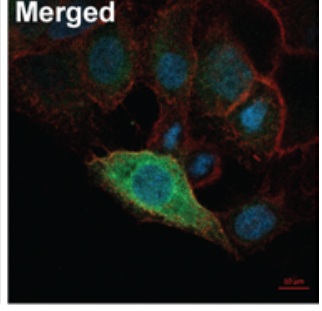

Merged

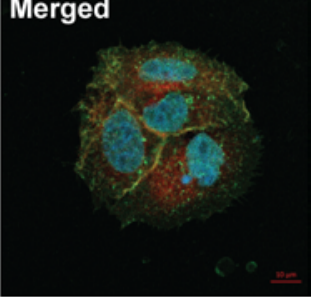

Merged

Figure 4. Tissue and cellular distribution of PRL2. (A)

$X$-gal-stained tissues of BAT

(left panels) and skeletal muscle (right panels) are shown. A blue stain indicates expression of $\mathrm{Pr} / 2$ mRNA in Prl2 heterozygote (Het) tissues. Tissues from WT were used as negative control. Scale bar: $10 \mu \mathrm{m}$. (B) BAT were stained with either no primary antibodies (left) or anti-CNNM3 antibodies (right). Signals were detected by Alexa Fluor 594-conjugated (red) anti-rabbit antibodies. Scale bar: $20 \mu \mathrm{m}$. (C) GFP-tagged PRL2 (green) and mCherry-tagged CNNM3 (red) were transiently overexpressed in HeLa cells. Alexa Fluor 488conjugated (green) or -594-conjugated (red) wheat germ agglutinin (WCA) were used to stain plasma membrane. Empty pCDNA3 plasmids were used for negative control. Scale bar: $10 \mu \mathrm{m}$.

Decreased Acly expression and increased citric acid cycle metabolite levels in PRL2-KO BAT. To gain a comprehensive view of the metabolic events in PRL2-KO BAT, mRNA expression levels of major metabolic genes were verified (Figure 6H). Among them, only ATP citrate lyase (Acly) expression was significantly decreased in both male and female PRL2-KO BAT (Figure 6H). As shown schematically in Supplemental Figure 5, ACLY is the enzyme that synthesizes cytosolic Acetyl-CoA from citrate. Therefore, citric acid 
A

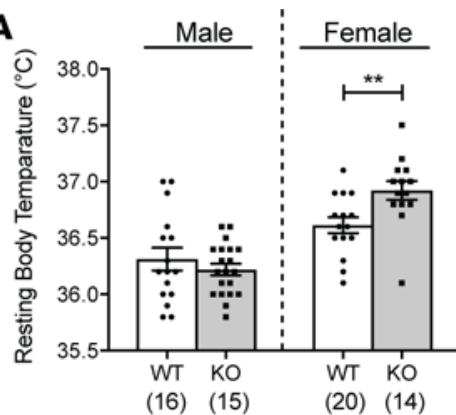

B

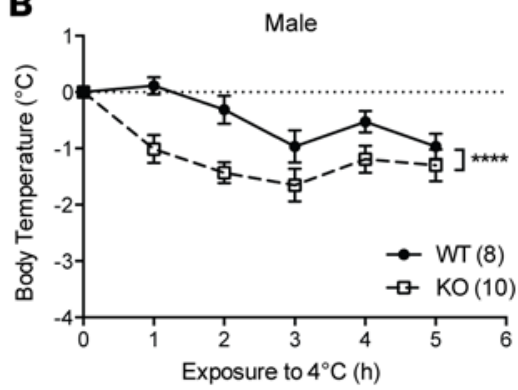

Female

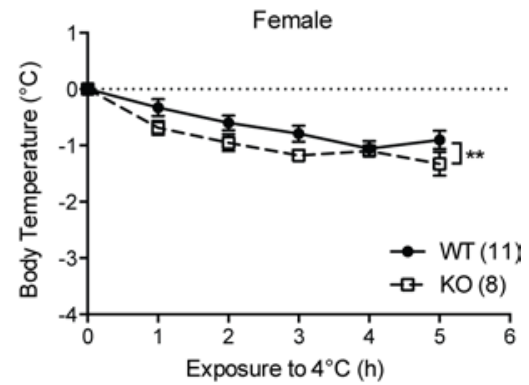

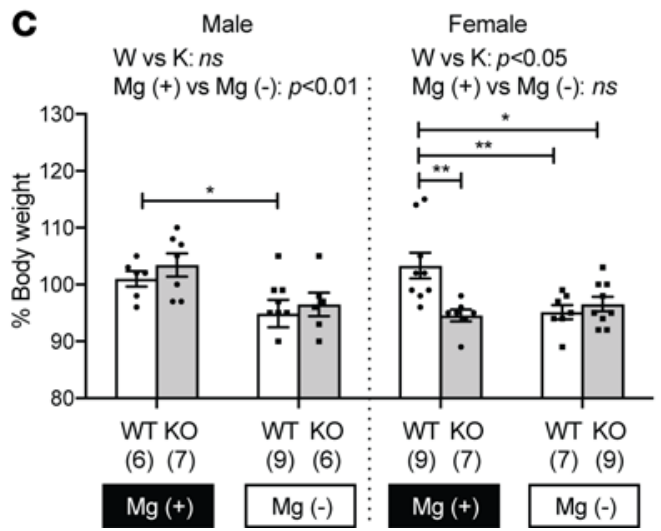

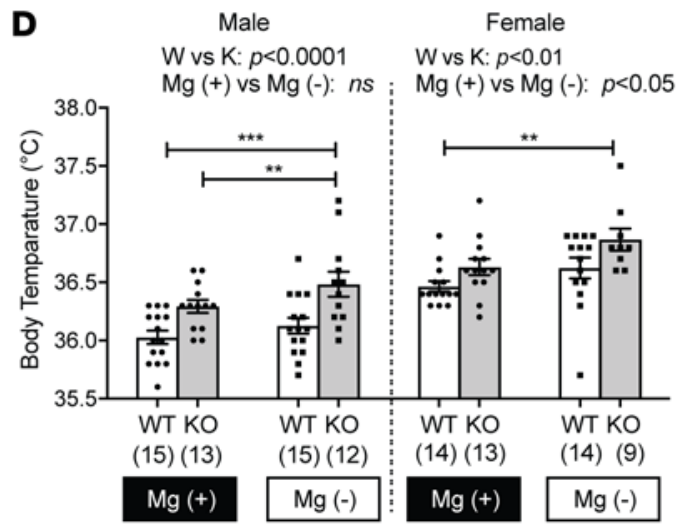

Figure 5. PRL2 deficiency sex-dependently altered thermogenesis. (A) Resting body temperature was measured using rectal thermocouple probes under normal housing temperature $\left(23^{\circ} \mathrm{C}\right)$. (B) Rectal body temperatures were measured after acute cold $\left(4^{\circ} \mathrm{C}\right)$ exposure. Data are presented as mean differences from the body temperature before cold challenge. (C) Percent body weight after a week of feeding control diets $\left.\left(\mathrm{Mg}^{[+}\right]\right)$or $\mathrm{Mg}^{2+}$-deficient diets (Mg [-]) is shown. Initial body weight at day 0 was set as $100 \%$. (D) Average core body temperature after a week of feeding control diets (Mg [+]) or Mg ${ }^{2+}$-deficient diets $(\mathrm{Mg}[-])$ is shown. Data is expressed as mean $\pm \mathrm{SEM}$. Number of animals analyzed is indicated in parenthesis in the figures. $P$ values were calculated by Student's $t$ test (A), two-way ANOVA (indicated statistical results are for WT vs. KO) (B), and two-way ANOVA with Tukey's multiple comparison test (C and D). ${ }^{*} P<0.05 ;{ }^{* *} P<0.01 ;{ }^{* *} P<0.001 ;{ }^{* * *} P<0.0001$.

cycle-related (CAC-related) metabolites including citrate were measured using gas chromatography-mass spectrometry (GC-MS) (Figure 6I). Notably, CAC metabolite levels were increased in PRL2-KO BAT (Figure 6I). These results suggest that loss of PRL2 inhibits the ACLY axis, which will lead to decrease fatty acid synthesis. Overall, this data indicates that PRL2 deficiency promotes catabolism through uncoupling reactions, while inhibiting anabolism as indicated by the regulation of the ACLY axis.

Circadian regulation of $P R L$ and CNNM gene expression. While circadian variations in serum and urinary $\mathrm{Mg}^{2+}$ have been reported $(23,24)$, the mechanisms underlying these rhythms and their biological importance remain poorly understood. A recent study identified a link between intracellular $\mathrm{Mg}^{2+}$ concentration and dynamic regulation of cellular energy balance over the circadian cycle and, importantly, those rhythmic changes in metabolism giving the feedback to control circadian gene expression (4). Since our results have shown a role for PRL2 in $\mathrm{Mg}^{2+}$ homeostasis and cellular metabolism, we hypothesized that PRL2 might regulate circadian energy metabolism by controlling the daily variation of $\mathrm{Mg}^{2+}$ flux. To assess its possible function in circadian rhythms, we first analyzed publicly available circadian datasets. Interestingly, all PRL

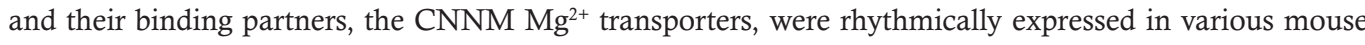
tissues (Table 2, Figures 7A and Supplemental Figure 6) (25-28) (CircaDB; http://circadb.hogeneschlab. org/) and clock transcriptional factors bound to most $\mathrm{Prl}$ and Cnnm promoters, in many cases with a rhythmic manner $(29,30)$ (Table 2 and Figure 7B). Period 1/2 (PER1/2) and cryptochrome (CRY1/2) binding rhythms are in antiphase to CLOCK/Neuronal PAS domain-containing protein 2:Brain and Muscle Arntlike Protein 1 (CLOCK/NPAS2:BMAL1), as would be expected given their roles as circadian repressors and activators, respectively (Figure 7B). It is noteworthy that rhythmic expression of Prl2 and Cnnm2 were found in BAT over the circadian cycle, and a daily expression pattern of Acly was paralleled with rhythmic PRL2 expression (Figure 7A) (26). The circadian expression of PRL1/2 and CNNM3 proteins in the liver 
Table 2. Circadian regulation of PRL and CNNM genes

\begin{tabular}{|c|c|c|c|c|c|}
\hline Human gene & Accession (protein) & $\begin{array}{c}\text { Circadian mRNA in mouse tissue }(P \\
<0.05)^{A}\end{array}$ & $\begin{array}{l}\text { Circadian TF binding } \\
\text { gene promoter }\end{array}$ & $\begin{array}{l}\text { Circadian TF binding outside } \\
\text { gene promoter }\end{array}$ & $\begin{array}{l}\text { U20S siRNA } \\
\text { knockdownc }\end{array}$ \\
\hline $\begin{array}{l}\text { Ptp4A1 } \\
\text { (Pr/1) }\end{array}$ & NP_003454 & $\begin{array}{l}\text { Liver, SCN, Lung, Kidney, Adrenal } \\
\text { gland, Heart, White adipose, } \\
\text { Pituitary, Hypothalamus, Brain } \\
\text { stem }\end{array}$ & $\begin{array}{l}\text { BMAL1, CLOCK, CRY1, } \\
\text { CRY2, REV-ERB } \beta^{\circ}\end{array}$ & $\begin{array}{c}\text { BMAL1 }(-66,585 \&-84,490), \\
\text { CLOCK(-66,580 \& -88,401), } \\
\text { PER1 }(-84,440 \&-69,830) \\
\text { PER2 }(-66,456,-84,261 \\
\&-69,715), \text { CRY1 }(-69,705 \\
-84,510, \&-65705), \text { CRY2 } \\
(-69,675 \&-66,515) \\
\text { CBP }(-65,740)\end{array}$ & Long period (1/4) \\
\hline $\begin{array}{l}\text { Ptp4A2 } \\
\text { (Prl2) }\end{array}$ & NP_001182029 & $\begin{array}{l}\text { Liver, Lung, SCN, Heart, Aorta, } \\
\text { Brown adipose, Kidney }\end{array}$ & $\begin{array}{l}\text { BMAL1, PER1, PER2, } \\
\text { CRY1, CRY2 }\end{array}$ & $\begin{array}{c}\text { BMAL1 }(-9,558), \text { PER1 }(34,802), \\
\text { PER2 }(34,792), \text { CRY1 }(34,752), \\
\text { CRY2 }(34,882,-12,688, \& \\
-15,158)\end{array}$ & Long period (3/4) \\
\hline $\begin{array}{l}\text { Ptp4A3 } \\
\text { (Prl3) }\end{array}$ & NP_009010 & Heart, Kidney & $\begin{array}{l}\text { Binding not detected } \\
\text { in mouse liver }\end{array}$ & $\begin{array}{c}\text { PER1 }(29,775), \text { CRY1 }(29,840), \\
\text { CRY2 }(29,740),\end{array}$ & $\begin{array}{l}\text { Long period (4/4), } \\
\text { Low amplitude (3/4) }\end{array}$ \\
\hline Cnnm3 & NP_060093 & $\begin{array}{l}\text { Kidney, Liver, Distal colon, Heart, } \\
\text { White adipose, Embryo fibroblast }\end{array}$ & $\begin{array}{l}\text { CRY1, CRY2 } \\
\text { REV-ERB } \beta^{\circ}\end{array}$ & & Long period (3/4) \\
\hline Cnnm4 & NP_064569 & $\begin{array}{l}\text { Kidney, Liver, Distal colon, Embryo } \\
\text { fibroblast, Skeletal muscle }\end{array}$ & $\begin{array}{l}\text { BMAL1, CLOCK, } \\
\text { NPAS2, CRY1 }\end{array}$ & & Short period (4/4) \\
\hline \multicolumn{6}{|c|}{$\begin{array}{l}\text { Results obtained from publicly available circadian datasets are shown. All known Prl and Cnnm genes are reported as their longest transcriptional } \\
\left.\text { isoform. AFrom CircaDB ( } 28) \text { with JTK_cycle } P<0.05 \text {. }{ }^{B} \text { From ChIP-Seq data in mouse liver ( } 29\right) \text {, defining a gene promoter as }-1200 \text { bp and }+50 \text { bp from the } \\
\text { transcriptional start site (TSS). Brackets indicate number of base pairs from TSS. 'From BioGPS (31, 63), whereby long period refers to }>25.5 \mathrm{~h} \text {, short } \\
\text { period refers to }<23.5 \mathrm{~h} \text {, and low amplitude refers to }<1,000 \text {. }{ }^{D} \text { From ChIP-Seq data in mouse liver }(64) \text {. Knockdown experiments used } 4 \text { different siRNA } \\
\text { constructions; fractions in brackets indicate the number of siRNAs that produced the indicated effect. }\end{array}$} \\
\hline
\end{tabular}

was further confirmed (Figure 7C). These data suggest that both $\mathrm{Prl}$ and Cnnm family genes are likely under the regulation of transcriptional activators CLOCK-BMAL1 and NPAS-BMAL1 and repressors PER-CRY and REV-ERBs. Moreover, siRNA knockdown of each $\mathrm{Prl}$ and $\mathrm{Cnnm}$ family gene, except $\mathrm{Cnnm4}$, led to longer circadian periods in U2OS cells (Table 2) (31). As Prl and Cnnm genes are circadian regulated, this suggests they might also bilaterally regulate cellular timekeeping.

Sex-dependent effects on metabolic and behavioral rhythms in PRL2-KO mice. It has been suggested that circadian clock genes drive cellular metabolism (32). Given our findings that Prl and Cnnm genes are under circadian regulation, we sought to determine whether the absence of PRL2 would alter circadian metabolic rhythms. Firstly, we found that daily food intake was significantly increased in female PRL2-KO mice (Figure 8A). We next measured basal energy expenditure and locomotor activities over 2 consecutive days using indirect calorimetry. Interestingly, although total day and night locomotor activity was comparable between PRL2-KO and WT mice (Figure 8B), daily rhythms of activity were altered in PRL2-KO mice (Figure $8 \mathrm{C}$ ). A phase shift of the diurnal activity rhythm, reduced amplitude, and lower baseline activity were observed in PRL2-KO males (Figure 8C, left panel). Meanwhile, we observed no phase shift of the rhythm but enhanced amplitude and higher baseline activity in PRL2-KO females (Figure 8C). In parallel with increased daily food intake, $\mathrm{O}_{2}$ consumption was also increased in female PRL2-KO mice, especially at night (Figure 8D). Additionally, the average respiration quotient (RQ) was increased in female PRL2-KO mice (Figure 8E). Male PRL2-KO mice exhibited comparable $\mathrm{O}_{2}$ consumption and RQ to WT mice (Figure 8, D and E). However, detailed analysis revealed that RQ was negatively correlated with body weight, even in male PRL2-KO mice (Figure 8F). The higher RQ observed in PRL2-KO mice indicated that relatively more carbohydrates were being oxidized as sources of energy compared with WT mice.

Similar to the phase shift observed in diurnal locomotor activity rhythms (Figure 8C, left panel), the phase of RQ rhythms were also shifted earlier in male PRL2-KO mice, and both baseline and amplitude 


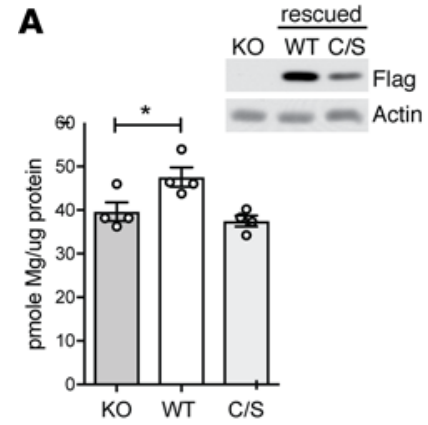

D Male/UCP1

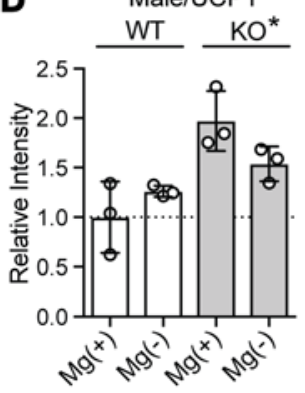

F

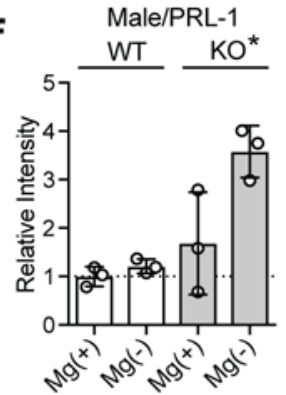

H

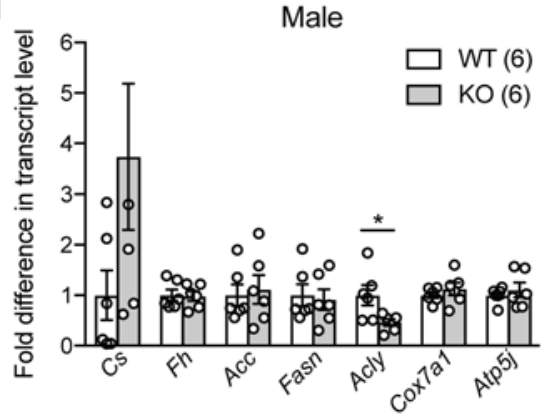

I

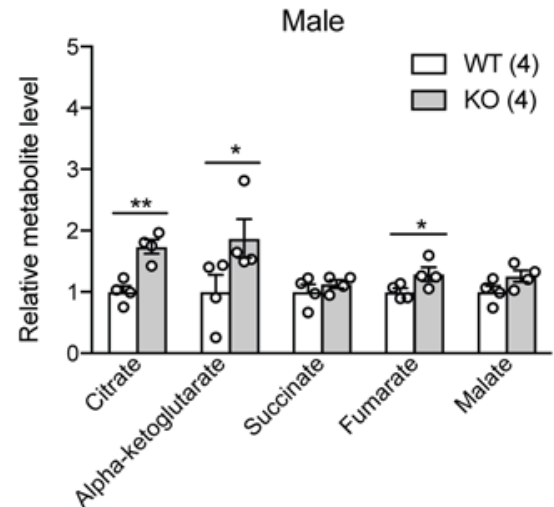

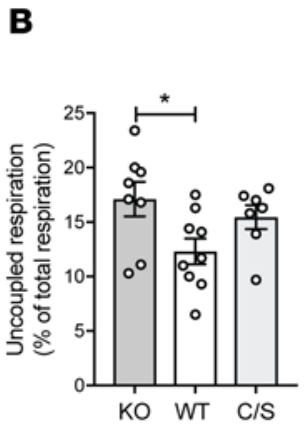
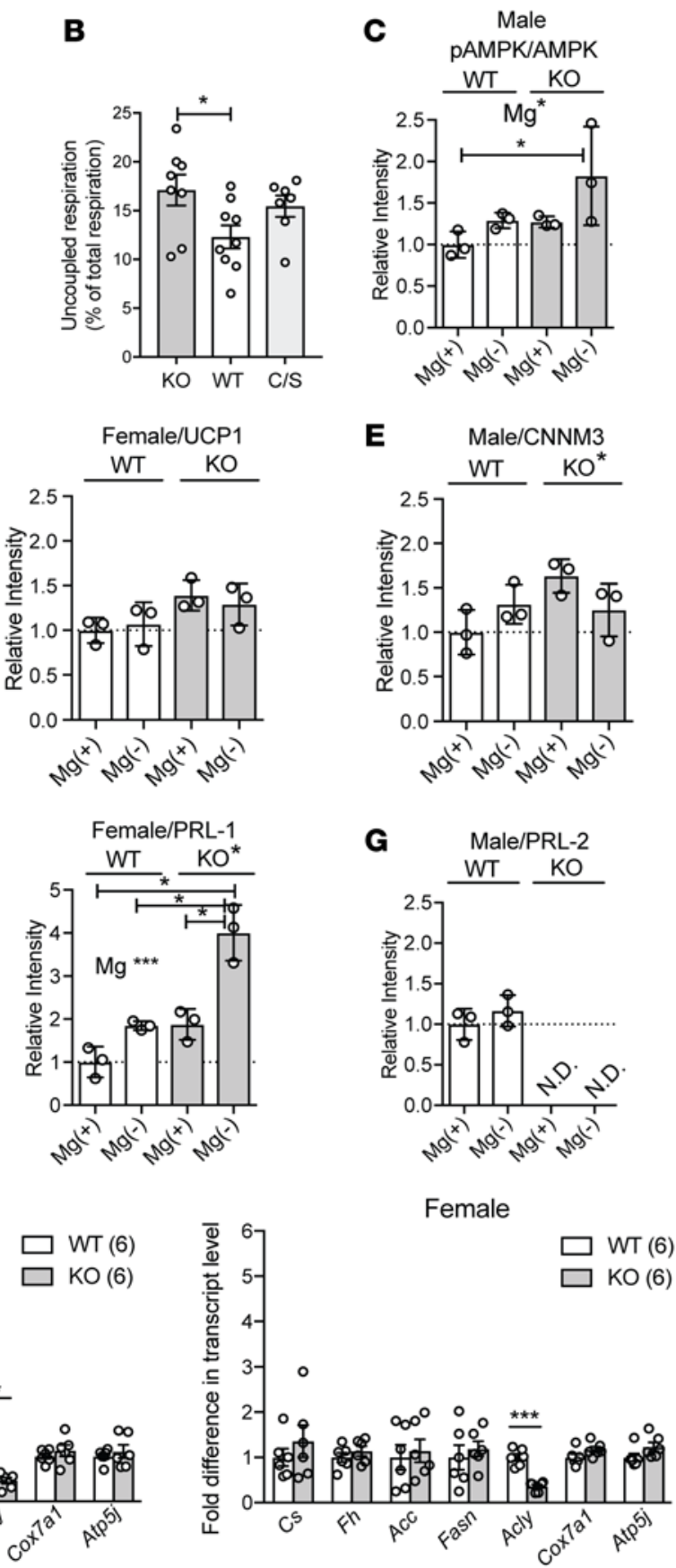

G
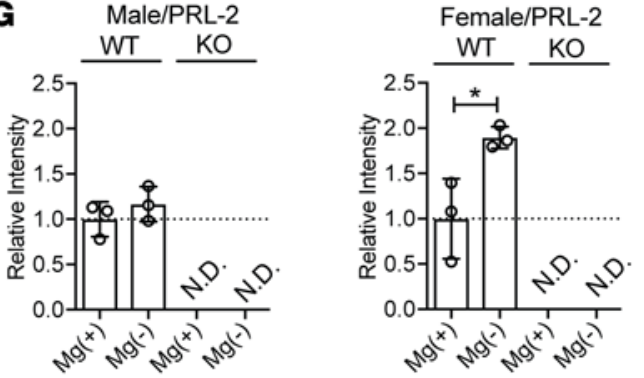

Female

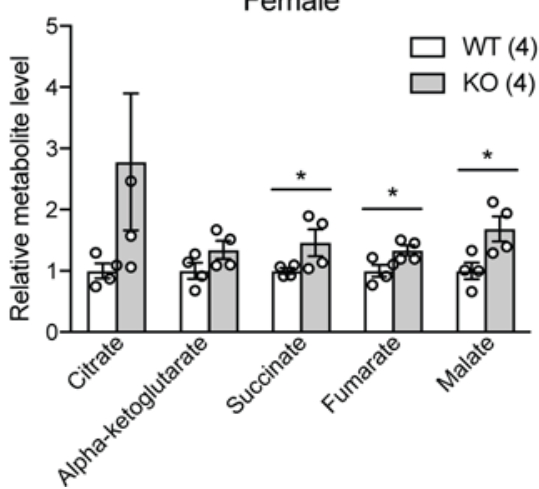


Figure 6. Altered cellular metabolism in PRL2-KO MEF and BAT. (A) $\mathrm{Mg}^{2+}$ content of immortalized PRL2-KO mouse embryonic fibroblasts (MEF) rescued with empty control (KO), Flag-PRL2 (WT), or Flag-PRL2 C101S (C/S) are shown. Expression of Flag-PRL2 transgene was immunoblotted with a Flag antibody. Actin antibody was used to confirm equal loading of lysates. (B) Fraction of cellular respiration devoted to uncoupled respiration of PRL2 (KO), C/S, or PRL2 (WT) MEF are shown. (C-C) Relative band intensity of the immunoblotting is shown. Protein lysates were obtained from 3 independent BAT samples after a week of feeding control diets $(\mathrm{Mg}[+])$ or $\mathrm{Mg}^{2+}$-deficient diets (Mg [-]). They were immunoblotted with indicated antibodies, quantified, and normalized against total protein using stain-free technology. Three individual animals were used for each condition. Data is expressed as fold change versus Mg (+) WT. ND, not detectable. (H) Quantitative PCR analyses were performed on RNA extracted from BAT. Data were normalized to $\beta$-actin and expressed as fold change versus WT. (I) Amounts of each metabolite in BAT were quantified by GC-MS. Data were normalized to internal standard myristic acid-D27 and sample weight and expressed as fold change versus WT. Data is expressed as mean $\pm \mathrm{SEM}(\mathbf{A}, \mathbf{B}, \mathbf{H}, \mathbf{I})$ and mean $\pm \mathrm{SD}$ (C-C). $P$ values were calculated by one-way ANOVA with Dunnett's multiple comparison (A and B), two-way ANOVA with Tukey's multiple comparison (WT vs. KO, indicated as $\left.\mathrm{KO}^{*}\right)\left(\mathrm{Mg}(+)\right.$ vs $\mathrm{Mg}(-)$, indicated as $\left.\mathrm{Mg}^{*}\right)(\mathbf{C}-\mathbf{F})$, Mann-Whitney test (C) and Student's $t$-test $(\mathbf{H}$ and $\mathbf{I})$. Two data points (9.03 and 7.23$)$ in KO-Cs are outside the axis. ${ }^{*} P<0.05 ;{ }^{* *} P<0.01 ;{ }^{* *} P<0.001$. Number of animals analyzed is indicated in parenthesis in the figures.

were reduced (Figure 8G, left panel). In contrast to males, female PRL2-KO mice showed no phase shift of the RQ rhythm and increased baseline but showed reduced amplitude (Figure 8G, right panel).

Taken together, our results show dysregulation of total energy metabolism in PRL2-KO mice in a sex-dependent manner, highlighting a key role for PRL2 in circadian rhythms of behavior and metabolism.

\section{Discussion}

Beyond previous studies that identified PRL2 functions in cellular $\mathrm{Mg}^{2+}$ homeostasis (8, 9), herein we demonstrate a requirement of PRL2 for sex-dependent and circadian rhythm-dependent energy metabolism (Figure 9 and Supplemental Figure 7). Since $\mathrm{Mg}^{2+}$ is a crucial rate-limiting factor for many metabolic effectors, we propose that differential PRL2 expression levels would regulate the concentration of intracellular $\mathrm{Mg}^{2+}$ to balance cellular energy requirement. Therefore, regulation of PRL2 expression through sex-dependent and circadian rhythm signals provides additional layers of global regulation on cellular metabolism via changes in intracellular $\mathrm{Mg}^{2+}$.

Genetic background, dietary $\mathrm{Mg}^{2+}$, and functional redundancy of PRL family protein. During the course of phenotypic analysis, sex differential phenotypes were first spotted as differences in neonatal lethality. As shown in Supplemental Figure 1C and Table 1, percentages of survival of PRL2-KO mice were significantly decreased in female on C57BL/6N background. Previously, Dong Y. et al. also reported lower body weight of newborn and adult PRL2-KO mice due to underdeveloped placenta (18). However, in their study, PRL2-KO mice were viable after birth (18). According to our results, phenotypes of PRL2-KO mice were susceptible to genetic background and $\mathrm{Mg}^{2+}$ contents in diets (Figure 5). Thus, the different neonatal mortality seen between these two studies might be attributed in part to differences in genetic background crossed (C57BL/6J vs. C57BL/6N) and in dietary $\mathrm{Mg}^{2+}$ contents. To protect themselves from hypothermia, BAT is proportionally abundant in newborn mammals. This suggest that neonates would be more sensitive to defective BAT functions, which may explain the higher neonatal lethality observed in PRL2-KO mice.

Since PRL1, PRL2, and PRL3 are highly homologous protein, and all PRLs bind to CNNMs (8, 9), they might have redundant physiological functions in animals. Indeed, recent study by Zhang's group strongly suggested functional redundancy between PRL1 and PRL2 during spermatogenesis (33). In our study, expression of PRL1 but not PRL3 were upregulated in PRL2-KO BAT (Figure 3, B and D, and Figure 6F), suggesting that PRL1 might partially compensate for PRL2 deficiency in BAT.

A sex-dependent expression of PRL2 in BAT. We observed that PRL2 deficiency differently affects energy metabolism between sexes (Supplemental Figure 6). It is common knowledge that overall metabolic activities are different between males and females, and thermogenic activities of BAT play important roles for sex-specific energy metabolism $(34,35)$. The main physiological function of BAT is nonshivering thermogenesis, which dissipates energy as heat to maintain body temperature (36). Consequently, BAT activity negatively correlates with the total amount of body fat, since nutritional energy is burned rather than stored as fat (37). BAT came under greater scrutiny following the discovery that these specialized adipocytes were present in adult humans (38-40), and BAT in women was shown to represent a larger mass with a higher glucose uptake activity relative to men (40-42). These results denote substantial differences in metabolic status between male and female BAT.

Although only female PRL2-KO mice exhibited higher body temperature with $\mathrm{Mg}^{2+}$-rich regular diets, when dietary $\mathrm{Mg}^{2+}$ was reduced, male PRL2-KO mice also exhibited higher body temperature (Figure 5, $\mathrm{A}$ and $\mathrm{D}$ ). This implies that intrinsic differences exist in thresholds for $\mathrm{Mg}^{2+}$ deficiency between male and 
A

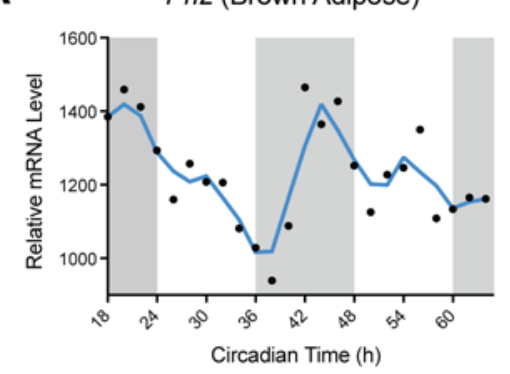

Cnnm2 (Brown Adipose)

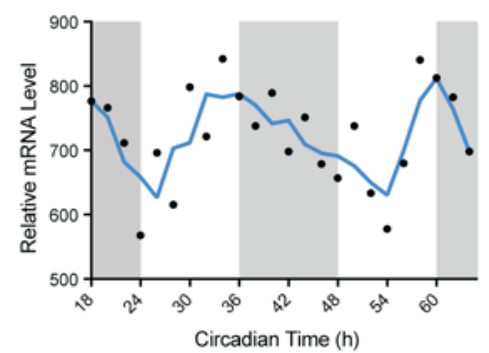

Acly (Brown Adipose)

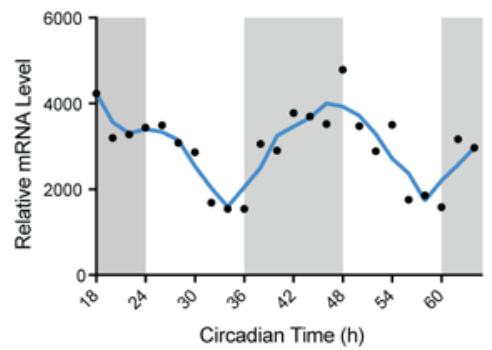

B

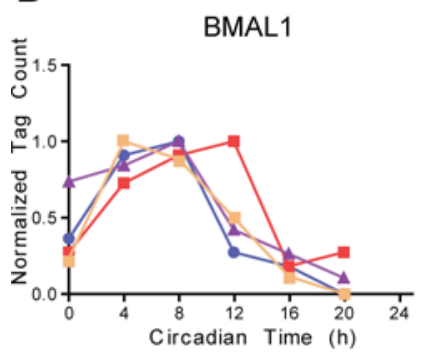

CRY1
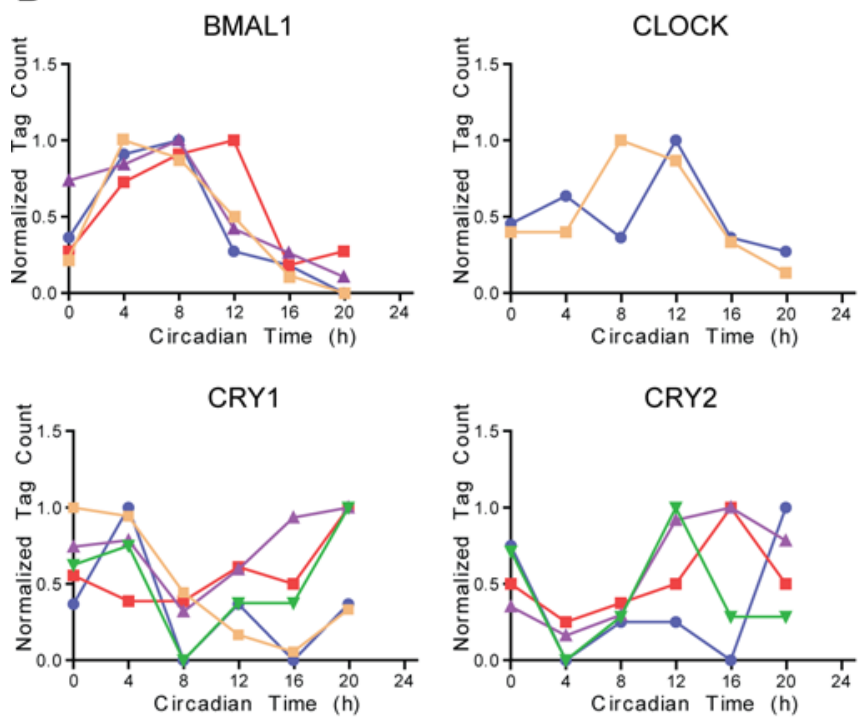

CRY2

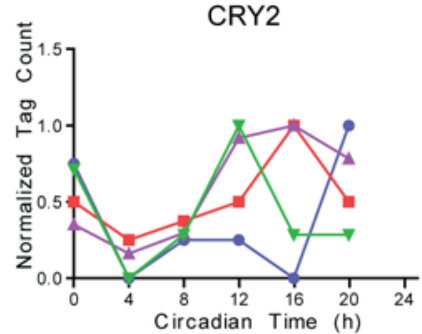

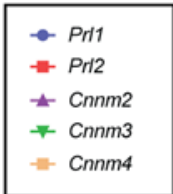

PER2
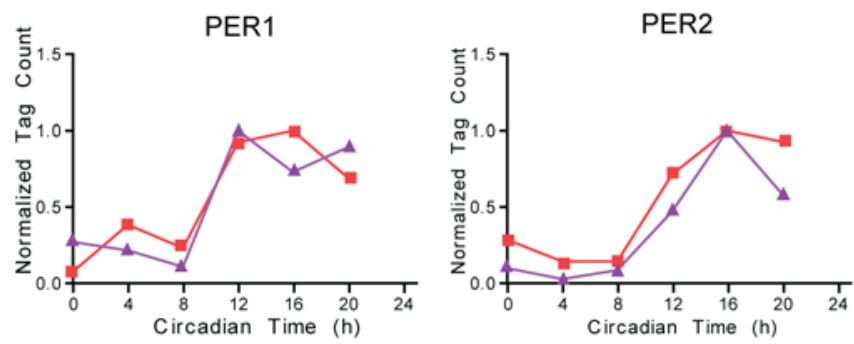

C

PRL1\&2 expression (Liver)

CNNM3 expression (Liver)
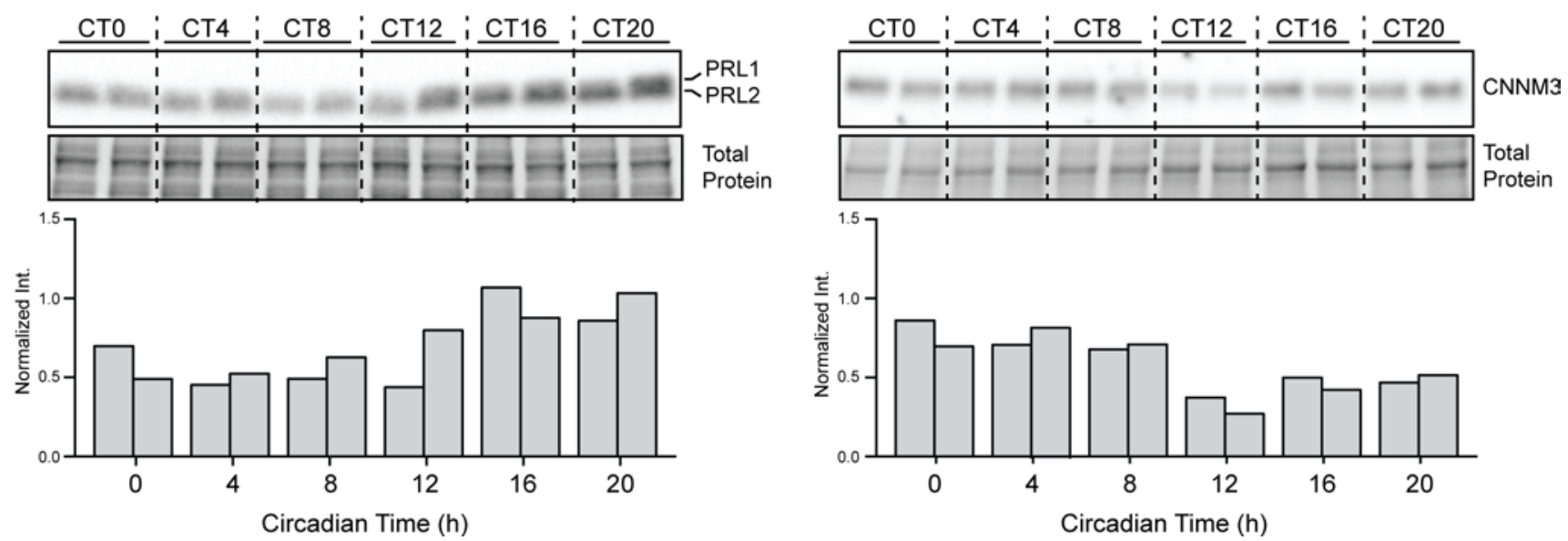

Figure 7. Circadian regulation of PRL and CNNM genes. (A) Relative mRNA levels of Prl2, Cnnm2, and Acly in BAT from publically available microarray data (26). (B) Binding affinity of core circadian transcription factors (TFs) to the Prl and Cnnm promoters throughout the circadian cycle. Tag counts were unity-normalized across the sampling interval, providing a rough indication of TF:DNA binding affinity throughout the circadian cycle. Circadian time (CT) 0-12 = subjective day, CT 12-24 = subjective night (30). (C) Western blot analysis of mouse liver lysates. Total protein extracts from 2 mice at each CT were loaded and immunoblotted for PRL1/2 and CNNM3 antibodies (upper panel). Signal intensity was quantified, and data were normalized against the stain-free total protein measurement (lower panel). 
A

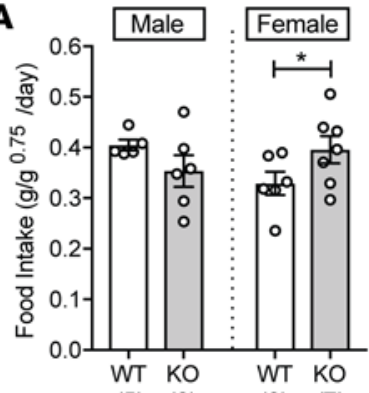

(5) (6) (6) (7)

C

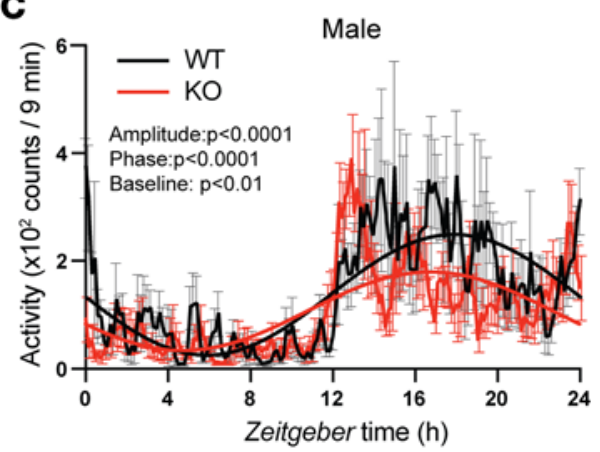

D

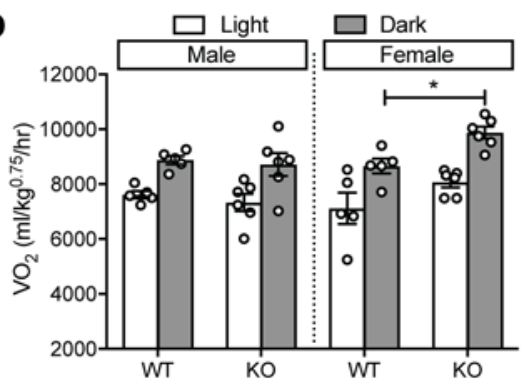

(5)

$\mathbf{F}$
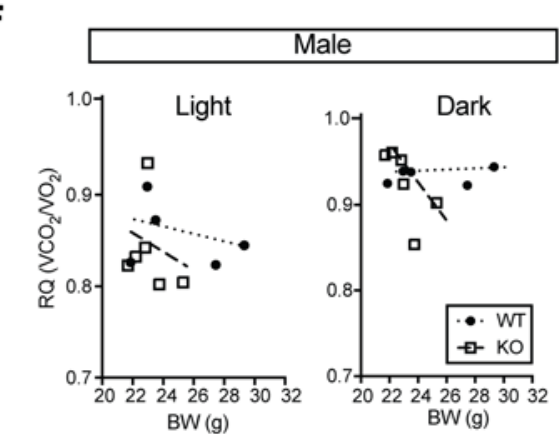

G

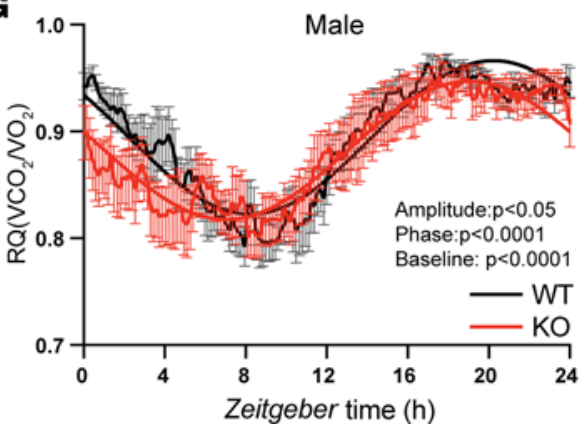

B

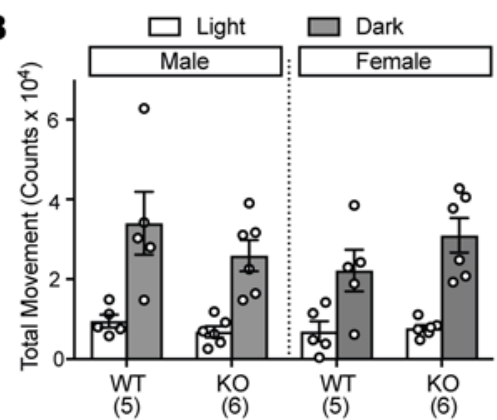

(5)

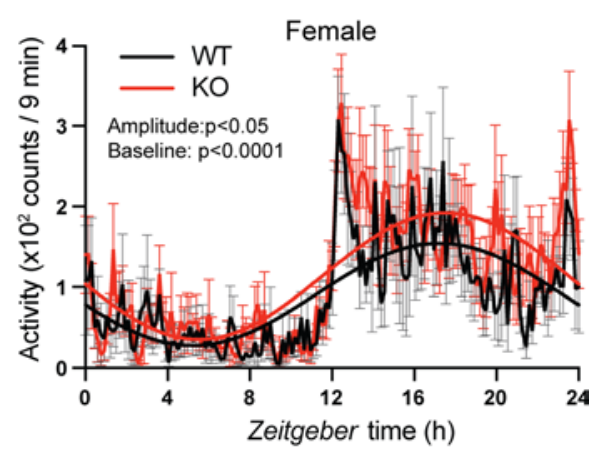

E

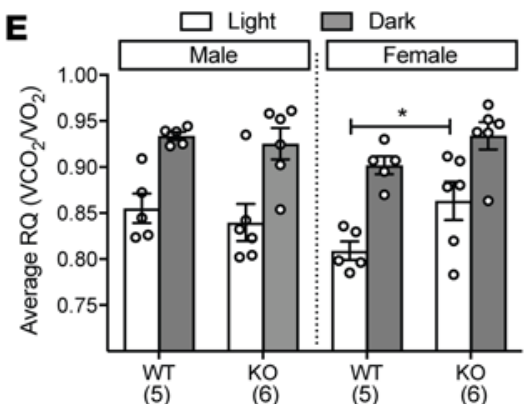

(5)
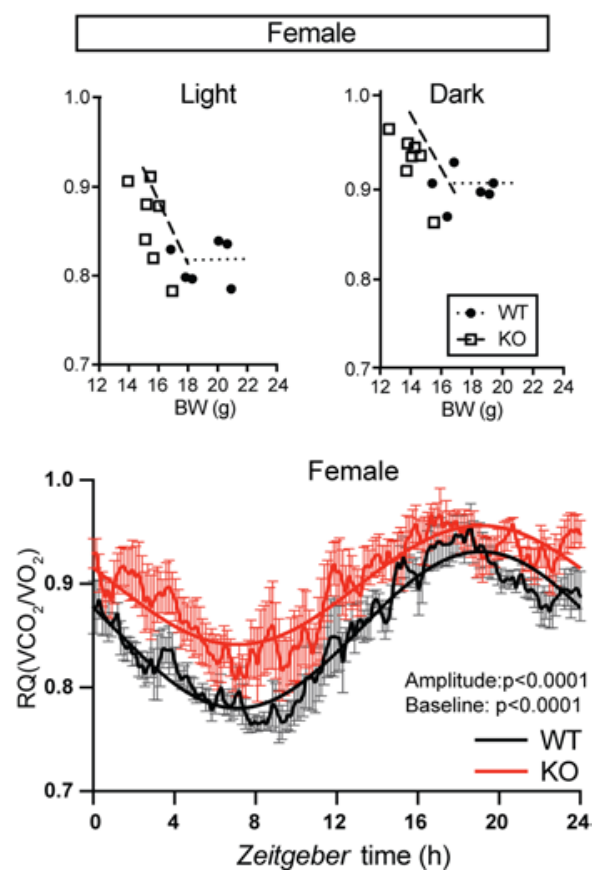

Figure 8. Sex-dependent metabolic and behavioral rhythms in PRL2-KO mice. (A) Average food intake per day is normalized by body weight with a power of 0.75 . (B) Total locomotor activity was counted as photobeam breaks. (C) Diurnal total locomotor activity accumulated within 9-min intervals. Lines indicate significant circadian cosine-wave fit. (D) Oxygen consumption $\left(\mathrm{VO}_{2}\right)$ was measured by indirect calorimetry for 48 hours. Average $\mathrm{VO}_{2}$ per hour during light and night phases was normalized by body weight with a power of 0.75. (E) Average $\mathrm{RQ}\left(\mathrm{VCO}_{2}\right.$ produced $/ \mathrm{VO}_{2}$ consumed) during light and night phases are shown. (F) Individual average RQ are plotted with body weight. Lines indicate linear regression. (C) Diurnal RQ rhythms are shown. Lines indicate significant circadian cosine-wave fit. Zeitgeber times 0 and 12 are the times of lights on and off, respectively. Data is expressed as mean \pm SEM. Number of animals analyzed is indicated in parenthesis in the figures. $P$ values were calculated by Student's $t$ test. Statistical significance was corrected for multiple comparisons using Holm-Sidak method (A, D, E). Circadian variation was tested by fitting a cosine wave equation on locomotor and RQ data, with a fixed 24-hour period; significance was determined by $F$ test ( $\mathbf{C}$ and $\mathbf{G}) .{ }^{*} P<0.05$.

female PRL2-KO mice. Similarly, WT mice showed higher body temperatures when they were fed a $\mathrm{Mg}^{2+}$-deficient diet, corroborating the requirement of $\mathrm{Mg}^{2+}$ for proper thermogenesis

Thermogenic activity of BAT is entirely UCP1 dependent; however, activity of UCP1 is constantly masked by purine nucleotides such as ATP under thermoneutral environment at around $30^{\circ} \mathrm{C}(36)$. It has been suggested that acute $\mathrm{Mg}^{2+}$ deficiency may unmask UCP1 activity in vivo, thus leading to increased proton leakage in BAT of $\mathrm{Mg}^{2+}$-deficient rats (43). Therefore, together with upregulation of UCP1 expression (Figure 6D), unmasking of UCP1 activity by $\mathrm{Mg}^{2+}$ deficiency could raise body temperatures in PRL2-KO mice.

In addition to BAT, skeletal muscle also plays a major role in energy metabolism, as it constitutes about $40 \%$ of body mass in mammals (44). Muscle shivering is the first line of defence against acute cold exposure, as these involuntary muscle contractions 


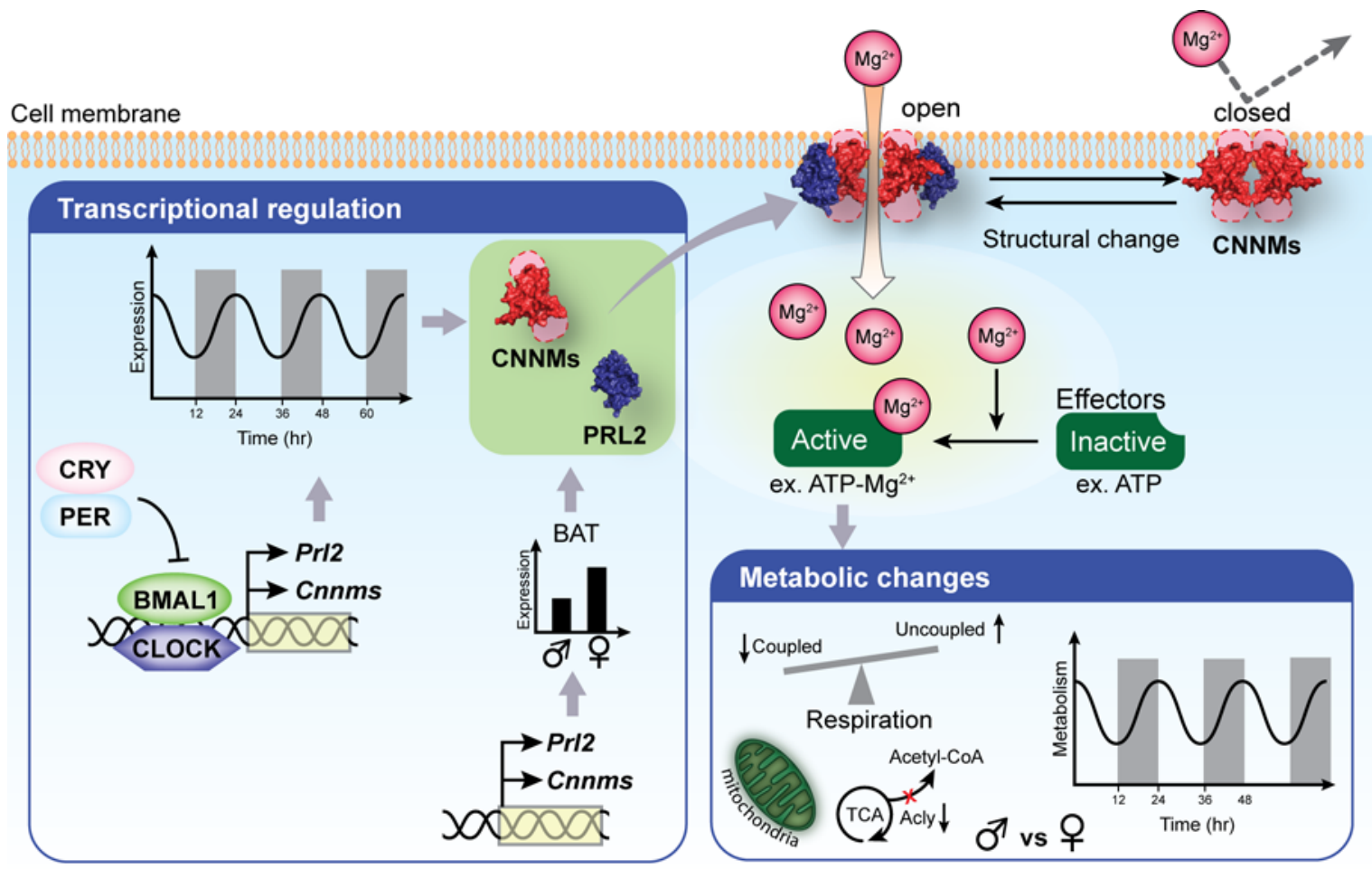

Figure 9. Graphical summary: PRL2 links magnesium flux and sex- and circadian-dependent energy metabolism.

produce heat rapidly by hydrolysing ATP (45). Our results showed that acute cold response was impaired in both male and female PRL2-KO mice (Figure $5 \mathrm{~B}$ ), indicating that the capacity of muscle shivering was diminished in PRL2-KO mice. It is interesting that defects in cold response were more evident in male PRL2-KO mice in comparison with female PRL2-KO mice, even when muscle weight is lower in female PRL2-KO mice. This inconsistency might be due to preexisting deficiencies in male PRL2-KO muscles that must compensate for the reduced BAT mass in order to maintain their body temperature. As observed in UCP1-ablated mice, defective BAT-dependent thermogenesis results in muscle exhaustion before cold exposure, which leads to an impaired cold response (45). Hence, it is likely that the defects we observed in acute cold response would relate to defects in BAT-dependent thermogenesis in PRL2-KO mice.

PRL2/CNNM complex, $\mathrm{Mg}^{2+}$, and cellular energy metabolism. PRL2-KO cells showed an increased ratio for uncoupled respiration, along with lower intracellular $\mathrm{Mg}^{2+}$ levels. These results support those in previous studies, which show that disruption of mitochondrial $\mathrm{Mg}^{2+}$ homeostasis reduces ATP production (46) and cellular $\mathrm{Mg}^{2+}$ deprivation decreases coupled respiration in cells (47). Notably, we and another group proposed that the PRL regulates energy metabolism by modulating intracellular $\mathrm{Mg}^{2+}$ through its association with the CNNM Mg${ }^{2+}$ transporter $(8,9)$. Based on this data, PRL2-KO cells appear to store less energy in the form of ATP and dissipate more energy due to defective $\mathrm{Mg}^{2+}$ flux. Thus, correlating with the enhanced BAT thermogenesis observed, the overall increase in energy dissipation can explain the reason PRL2-KO mice are underweight, even though they exhibited increased food intake and $\mathrm{O}_{2}$ consumption, especially in female mice.

$\mathrm{Mg}^{2+}$ transporter CNNMs contain tandem pairs of cystathionine- $\beta$-synthase (CBS) domains that are conserved in all living cells from prokaryotes to eukaryotes $(48,49)$. CBS domains act as energy-sensing modules; for instance, the $\gamma$ subunit of AMPK possesses 4 CBS domains with allosteric binding sites for AMP as well as ATP, allowing AMPK activity to vary according to the intracellular ATP:AMP ratio (50). Such energy-sensing mechanisms could modulate PRL2/CNNM functions, as ATP binds to CBS domains of CNNM2 in a $\mathrm{Mg}^{2+}$-dependent manner (51) and adenosine nucleotides trigger a conformational change in the CBS domains of CNNM2 (52). Taking into account that PRLs directly interact with CBS domains of CNNMs $(8,53)$, these findings imply that the function of the PRL2/CNNMs complex must be tightly regulated according to cellular metabolic status. 
PRLs and circadian rhythms. Recently, Feeney et al. reported a significant role for $\mathrm{Mg}^{2+}$ in the maintenance of the circadian rhythm-dependent metabolism that was conserved throughout evolution (4). This function in living cells involves the circadian regulation of hundreds of metabolic enzymes. The trigger for the $\mathrm{Mg}^{2+}$ cycling levels was, however, not identified. On this important question, the newly discovered PRL2 function that we report in circadian rhythms of behavior and metabolism is of high interest. As we have found in publicly available datasets, the expression of $\mathrm{Prl}$ and $\mathrm{Cnnm}$ family genes presents circadian regulation in various mouse tissues. Circadian oscillation of Prl2 expression was also found in the suprachiasmatic nuclei, the central clock, which coordinates circadian rhythms across the entire body (54) (Table 2). This suggests possible pivotal functions of PRL2 in circadian rhythm, linking clock genes and the circadian-regulated functions of $\mathrm{Mg}^{2+}$ in cells and organs. Indeed, PRL2-KO mice showed alterations in both locomotion activity and modified RQ rhythms (Figure 8, C and G).

Taken together, these results suggest a model whereby diurnal oscillation of PRL2 expression generates rhythmic $\mathrm{Mg}^{2+}$ fluxes, leading to proper daily cycles of metabolism. Moreover, as reported in Table 2, downregulation of PRL2 and CNNM1-4 were shown to affect circadian period (31). As observed by Feeney et. al., daily oscillation of intracellular $\mathrm{Mg}^{2+}$ provides a cell-autonomous timekeeping component that gives a cue to reset the cellular clock (4). Thus, based on the established role of PRL2 in $\mathrm{Mg}^{2+}$ homeostasis, one of the crucial PRL2 functions could be related to circadian timekeeping.

Concluding remarks. To our knowledge, our results identify a novel physiological function for PRL2 in circadian energy metabolism by linking key components of the circadian clock, PRL2, and intracellular $\mathrm{Mg}^{2+}$ to diurnal metabolic oscillations. By establishing PRL2 as part of the molecular mechanisms underlying intracellular $\mathrm{Mg}^{2+}$ fluctuations, we believe that physiological functions of PRL2 are rather broad and largely dependent on cellular context via pleiotropic actions of $\mathrm{Mg}^{2+}$ in cells. As growing evidence suggests that dysregulation of Prl2 or clock genes promote breast cancer tumorigenesis $(55,56)$, further investigations into the mechanisms regulating the Prl genes, circadian rhythms, and oncometabolism will be of key importance.

\section{Methods}

Generation of PRL2-KO mice, genotyping, and colony maintenance. The Prl2 gene-trapped ES cells (clone AQ0673, 129P2/OlaHsd, Sanger Institute GeneTrap Resource) were injected into blastocysts to generate chimera mice, and male chimeras were mated with $\mathrm{C} 57 \mathrm{Bl} / 6 \mathrm{~N}$ females (Harlan Laboratories). Germline-transmitted Prl2 heterozygous mice were kept as mixed genetic background $(129 \times \mathrm{B} 6)$, or they were backcrossed for 6 generations to C57BL/6N (Harlan Laboratories). Genotypes were determined by PCR using mixture of 3 primers; forward 1: 5' - AACAGTTAGATCAGCACCCAGGGTTAGG - 3', reverse 2: 5' - GCGCACTGGCTTGGTGAAATTTAGAAGA - 3', and reverse 3: 5' - TGGGGGTGGTGATATAAACTTGAGGCTG $-3^{\prime}$. Genomic DNA extracted from tail lysate were used as a template, and PCR was carried out $95^{\circ} \mathrm{C}$ for 120 seconds (s), followed by 30 cycles at $95^{\circ} \mathrm{C}$ for $45 \mathrm{~s}, 60^{\circ} \mathrm{C}$ for $60 \mathrm{~s}$, and $72^{\circ} \mathrm{C}$ for $80 \mathrm{~s}$. PRL2-KO mice were generated by intercrossing heterozygous parents with WT littermates used as controls. Eight- to 14-week-old mice were used for experiments unless specified in the manuscript. Mice were fed 2920X Teklad global soy protein-free extruded rodent diets $(0.2 \% \mathrm{Mg}$ by weight) (Harlan Laboratories) regularly, and $500 \mathrm{ppm}(0.05 \%$ by weight) Mg diets (TD.10654, Harlan Laboratories) and Mg deficient diets (TD.10652, Harlan Laboratories) were fed when indicated in the manuscript. Mice were kept under pathogen-free conditions in an environmentally controlled room (12-hour light:12-hour dark [LD] cycle, with lights on at 7 am, room temperature at $23^{\circ} \mathrm{C}$ ) at the Animal Resource Centre of McGill University. Circadian profiles of PRL1/2 and CNNM3 proteins were performed on liver samples collected from 2- to 3-month-old male C57BL/6J mice kept at the Douglas Mental Health University Institute. This was done at the indicated circadian times (CTs) on the second day in constant darkness, following entrainment in LD. CT0 and CT12 correspond to the times lights were on and off, respectively, in the prior LD cycle.

Resting body temperature and cold-exposure studies. Mice were housed individually with wood flake bedding and acclimated for 48 hours prior to the experiments. Resting body temperature was recorded between 12:00 pm to 1:00 pm each day for 5 days using an electric thermometer (TH-5, Physitemp) with rectal probe (RET-3, Physitemp). We considered a mouse "resting" when sleeping, and thus, the data were excluded when a mouse was apparently awake and moving at the time of recording. Average body temperature for an individual mouse was used as the resting body temperature. For cold-exposure studies, the mice were transferred to a $4^{\circ} \mathrm{C}$ cold room from normal housing temperature at $23^{\circ} \mathrm{C}$. Body temperature was recorded using a rectal probe before and every hour after $4^{\circ} \mathrm{C}$ cold exposure. 
Body compositions, adipocyte size, lipid droplet area, and muscle fiber size. Mice were euthanized by carbon dioxide, and nose to anus body lengths were measured on a flat surface. Tissues were dissected and weighted. WAT, BAT, and gastrocnemius muscle (GA) were fixed in 10\% phosphate buffered formalin (Fisher scientific) overnight, and H\&E-stained paraffin sections were prepared by standard procedure. Histological images were captured by Aperio ImageScope (Leica Biosystems). Adipocyte size, lipid droplet area, and muscle fiber size were measured using ImageJ (57).

$R N A$ isolation, reverse transcription, and quantitative PCR. Tissues were harvested from mice euthanized by carbon dioxide and immediately frozen in liquid nitrogen and stored at $-80^{\circ} \mathrm{C}$. Total RNA was extracted from frozen tissues using TRIzol (Invitrogen) according to manufacturer's instructions. To remove contaminated genomic DNA completely, extracted RNA was treated with DNase I (RiboPure RNA extraction kit, Invitrogen) at $37^{\circ} \mathrm{C}$ for 30 minutes, and DNase I was inactivated by adding DNase I inhibition beads for 2 minutes at room temperature. For assessment of Prl3 and Cnnms expression, $1 \mu \mathrm{g}$ of extracted RNA was used for synthesizing cDNA with SuperScript III reverse transcriptase (Invitrogen) according to manufacturer's instructions. Quantitative PCR (qPCR) was performed on a LightCycler 480 (Roche Life Science) with SYBR Green Master Mix (Roche Life Science) according to manufacturer's instructions. Actin gene was used as a reference gene to calculate the relative abundance of the indicated genes of interest. Primers for $C n n m$ family genes (Cnnm2-1, Cnnm2-2, Cnnm3-1, Cnnm3-2, and Cnnm4) were described in ref. 58. For assessment of metabolic gene expression, $1 \mu \mathrm{g}$ of total RNA from BAT was reverse transcribed using the iScript cDNA synthesis kit (Bio-Rad) following the manufacturers recommendations. qPCR was performed on the MyiQ2 Real-Time PCR detection system using the SYBR green master mix (Bio-Rad). Tbp was used as reference gene. Amplification of murine targets was done using specific primers found in the Supplemental Data.

Western blotting. Tissues were harvested from mice euthanized by carbon dioxide. Dissected tissues were immediately frozen in liquid nitrogen and stored at $-80^{\circ} \mathrm{C}$. Tissues were lysed and homogenized with RIPA buffer (50 mM Tris-HCl [pH 7.5], $150 \mathrm{mM} \mathrm{NaCl}, 1 \%$ NP-40, 0.5\% sodium deoxycholate, $0.1 \%$ SDS) supplemented with proteinase inhibitors (Roche Diagnostics, cOmplete). Tissue extracts were cleared by centrifugation at $16,000 \mathrm{~g}$ for 10 minutes at $4^{\circ} \mathrm{C}$. Protein concentration was determined using BCA assay kit (Pierce), as per the manufacturer's instructions. Western blot analysis was performed according to standard procedures using mouse monoclonal anti-PRL2 (EMD Millipore, 05-1583), rabbit polyclonal anti-CNNM3 (Proteintech, 13976-1-AP), rabbit polyclonal anti-UCP1 (Abcam, ab10983), and rabbit polyclonal anti- $\beta$-actin (Sigma-Aldrich, A2066) antibodies. Rabbit polyclonal anti-calnexin (2433), rabbit monoclonal anti-phosphor-AMPK $\alpha$ (T172; 5256), and rabbit polyclonal anti-AMPK $\alpha$ (2532) antibodies were obtained from Cell Signaling Technology. Intensity of the signal was quantified by the ImageJ (57) for Figure 3B or by the stain-free technology (Bio-Rad) for Figure 4C.

$X$-gal tissue staining and immunofluorescence. BAT and gastrocnemius muscles were dissected from 9-weekold PRL2 heterozygous and WT mice for X-gal tissue staining, and BAT was dissected from 12-week-old WT female C57BL/6N mice for immunofluorescence. Tissues were freshly embedded into Tissue-TekT compound (Sakura Finetek) and frozen using acetone/dry ice. Cryosections were prepared at 20- $\mu \mathrm{m}$ thickness for X-gal staining and 10- $\mu \mathrm{m}$ thickness for immunofluorescence. X-gal tissue staining was performed as described previously (17). For immunofluorescence, frozen sections were fixed with ice-cold methanol for 10 minutes. After blocking, sections were incubated with rabbit polyclonal anti-CNNM3 antibodies (1:30; Novus Biologicals, NBP1-90272) at $4^{\circ} \mathrm{C}$ overnight. Secondary detection was done using anti-rabbit secondary antibodies labeled with Alexa Fluor 594 (1:500; ThermoFisher Scientific, A11012). Fluorescent images were captured with a Zeiss LSM 800 confocal microscope (Carl Zeiss).

Subcellular localization of PRL2 and CNNM3. pCNNM3-mCherry plasmid was generated by cloning fused human CNNM3 (8) and mCherry cDNA into EcoRV and NotI sites of pcDNA3.1/Zeo(+) (ThermoFisher Scientific). Human PRL2 was cloned in pDONR221 as described previously (8) and shuttled into pDEST53 destination vector (ThermoFisher Scientific) according to the Gateway system protocol to generate the GFP N-terminal-tagged PRL2. HeLa cells were cultured on the 8-well Thermo Scientific Nunc Lab-Tek II Chamber Slide and transfected pGFP-PRL2 and/or pCNNM3-mCherry plasmids using Lipofectamine 2000 (ThermoFisher Scientific). Twenty-four hours after transfection, cells were fixed with phosphate buffered $10 \%$ formaldehyde for 10 minutes. When appropriate, cells were subsequently stained with Alexa Fluor 488 or 594 conjugated wheat germ agglutinin (ThermoFisher Scientific, W11261 and W11262, respectively) according to manufacturer's standard procedures. Fluorescent images were captured with a Zeiss LSM 800 confocal microscope (Carl Zeiss). 
Primary MEFs (PMEFs). PMEFs were isolated from E14.5 embryos according to the standard protocols. The cells were kept for at least 20 serial passages to immortalize spontaneously, and established PRL2-KO MEF lines cells were stably infected by retroviral infection using pMSCVpuro vector (Clontech) alone and with the vector containing the Flag-PRL2 (59). Following infection, cells were selected with $4 \mu \mathrm{g} / \mathrm{ml}$ puromycin for 2 weeks, and the resistant population was used for cellular assays.

Cellular respiration. Cellular respiration was measured using a Clark-type oxygen electrode as described previously (60).

$G C-M S$. Murine BAT was grinded in liquid nitrogen using mortar and pestle. Carefully weighted (10$20 \mathrm{mg}$ ) tissue powder was collected in prechilled tubes on dry ice. Metabolites were extracted using $600 \mu \mathrm{l}$ of a dry-ice cold $\mathrm{MeOH}$ :chloroform (2:1) solution. Following addition of 5 ceramic beads $(2.8 \mathrm{~mm}$, VRW 19-646-3), tubes were vortexed at high speed for 1 minute, followed by 10-minute sonication at a high setting with $30 \mathrm{~s}$ "on" and $30 \mathrm{~s}$ "off" cycles (Biorupter, Diagenone). Samples were vortexed again for $30 \mathrm{~s}$ and further sonicated for 5 minutes. Chloroform ( $200 \mu 1)$ and $200 \mu \mathrm{l}$ HPC grade water were added to samples followed by virougous shaking and resting for 10 minutes on ice. Samples were cleared by centrifugation at 21,000 $\mathrm{g}$ for 10 minutes at $4^{\circ} \mathrm{C}$; upper phase was kept and lower organic phase was discarded. Internal standard myristic acid- $\mathrm{D}_{27}(1 \mu \mathrm{l} ; 800 \mathrm{ng} / \mu \mathrm{l}$ dissolved in pyridine) was added to the tubes, which were subsequently evaporated until complete dryness (Laconco CentriVap concentrator). Sample methoximation, derivatization with MTBSTFA, GC-MS installations, and CAC metabolites identification features were previously described (61). Metabolite integration from $\mathrm{m}+0$ ions were divided by myristic acid- $\mathrm{D}_{27}$ integration and by sample weight.

Metabolic cage analysis. Oxymax Lab Animal Monitoring System: CLAMS (Columbus Instruments) was used to measure and analyze oxygen consumption, carbon dioxide production, and locomotor activity. The data were collected every 9 minutes. An 8- to 10-week-old mice was placed in an individual metabolic cage unit and acclimatized for 24 hours with food and water ad libitum. Measurements were taken for the subsequent 48 hours, and data were analyzed separated into dark and light phases. The data for oxygen consumption and carbon dioxide production were normalized by body weight with the power of 0.75 (62). To gauge food intake, 8 to 10 -week-old mice were placed in individual cages with wood flake bedding. Pellet foods $(10-20 \mathrm{~g})$ were given per mouse every day. Weight of the pellet foods and body weight were measured between 12:00 pm to 1:00 pm every day for 5 days. Average food intake per day and body weight were used to calculate food intake per day per body weight with the power of 0.75 . Locomotor activity data and metabolic data (respiratory quotient or $\mathrm{RQ}, \mathrm{VCO}_{2} / \mathrm{O}_{2}$ ) were analyzed over 48 hours using the ClockLab software (Actimetrics).

Statistics. Data is expressed as mean \pm SEM with the exception of data for Western blotting, which is reported as mean \pm SD. GraphPad Prism (GraphPad software) and Excel (Microsoft) were used to perform statistical analysis. Statistical method used to calculate $P$ values are indicated in figure legends. Circadian variation was tested by fitting a cosine wave equation: $y=$ Baseline $+($ Amplitude $\times \cos [(2 \times \pi)([x-$ Phaseshift]/24)]) on locomotor and RQ data, with a fixed 24-hour period; significance was determined by $F$ est (Figure $8, \mathrm{C}$ and $\mathrm{G}$ ). The $P$ values $<0.05$ were considered significant.

Study approval. All animal procedures were approved by the McGill Animal Care Committee (Montreal, Quebec, Canada) or the Douglas Institute Facility Animal Care Committee (Montreal, Quebec, Canada) and were conducted according to the Canadian Council of Animal Care ethical guidelines for animal experiments.

\section{Author contributions}

NU, SH, and MLT conceptualized the project. NU, SH, SPG, NNW, and VC investigated. Formal Analysis was done by NU, AP, and SK. Resources were handled by SK. NU wrote original draft. NU, SH, SPG, SK, AP, NC, JSP, and MLT reviewed and edited manuscript. Visualization was done by NU. NC, JSP, and MLT supervised. Funding acquisitionwas done by JSP, NC, and MLT.

\section{Acknowledgments}

The authors thank Élie Kostantin for constructing pCNNM3-mCherry expression plasmid vectors and Jeremy Gungabeesoon and Eva Mignon for technical help for maintaining mouse colony and helpful discussions. The authors also thank Raman Agnihotram for helping perform statistical analysis. MLT is a Jeanne and Jean-Louis Lévesque chair in Cancer Research. Microscopy images were collected and analyzed at the McGill University Life Sciences Complex Advanced BioImaging Facility (ABIF). 
Metabolite measurements were performed at the Rosalind and Morris Goodman Cancer Research Centre Metabolomics Core Facility supported by the Canada Foundation for Innovation, The Dr. John R. and Clara M. Fraser Memorial Trust, the Terry Fox Foundation (TFF Oncometabolism Team grant 116128 and 242122), and McGill University. This research is supported by an operating grant from Canadian Institutes of Health Research (CIHR): The PRL-CNNM-Mg ${ }^{2+}$ cascade, a new paradigm in oncogenesis: CIHR MOP 142497 to MLT. This work is also supported by the grant from Merck, Sharp \& Dohme Corp./McGill Faculty of Medicine Grant for Translational Research program: Characterization of the PTP4A2/CNNM3 Complex as a Novel Target for Cancer Therapy, no. PT 66481, and the Quebec Breast Cancer Foundation and the Canadian Breast Cancer Research Alliance, 020303 to MLT, as well as operating grants from the CIHR MOP-119322 and the Natural Science and Engineering Research Council N249731-2012 to NC. JSP is a Fonds de recherche du Québec - Santé (FRQS) Research Scholar, and this research is supported by an operating grant CIHR MOP-106603 to JSP.

Address correspondence to: Michel L. Tremblay, Rosalind and Morris Goodman Cancer Research Centre, McGill University, Cancer Research Building Room 617, 1160 Pine Avenue West, Montreal, Quebec, Canada H3A 1A3. Phone: 514.398.7290; Email: michel.tremblay@mcgill.ca.

1. de Baaij JH, Hoenderop JG, Bindels RJ. Magnesium in man: implications for health and disease. Physiol Rev. 2015;95(1):1-46.

2. Laires MJ, Monteiro CP, Bicho M. Role of cellular magnesium in health and human disease. Front Biosci. 2004;9:262-276.

3. Rubin $\mathrm{H}$. The paradox of the contrasting roles of chronic magnesium deficiency in metabolic disorders and field cancerization. Magnes Res. 2014;27(3):94-102.

4. Feeney KA, et al. Daily magnesium fluxes regulate cellular timekeeping and energy balance. Nature. 2016;532(7599):375-379.

5. Dunlap JC, Loros JJ. Yes, circadian rhythms actually do affect almost everything. Cell Res. 2016;26(7):759-760.

6. Hardy S, Julien SG, Tremblay ML. Impact of oncogenic protein tyrosine phosphatases in cancer. Anticancer Agents Med Chem. 2012;12(1):4-18.

7. Rios P, Li X, Köhn M. Molecular mechanisms of the PRL phosphatases. FEBS J. 2013;280(2):505-524

8. Hardy S, et al. The protein tyrosine phosphatase PRL-2 interacts with the magnesium transporter CNNM3 to promote oncogenesis. Oncogene. 2015;34(8):986-995.

9. Funato Y, Yamazaki D, Mizukami S, Du L, Kikuchi K, Miki H. Membrane protein CNNM4-dependent Mg2+ efflux suppresses tumor progression. J Clin Invest. 2014;124(12):5398-5410.

10. Kostantin E, et al. Inhibition of PRL-2.CNNM3 Protein Complex Formation Decreases Breast Cancer Proliferation and Tumor Growth. J Biol Chem. 2016;291(20):10716-10725.

11. Alonso A, et al. Protein tyrosine phosphatases in the human genome. Cell. 2004;117(6):699-711.

12. Zeng Q, Si X, Horstmann H, Xu Y, Hong W, Pallen CJ. Prenylation-dependent association of protein-tyrosine phosphatases PRL-1, -2, and -3 with the plasma membrane and the early endosome. J Biol Chem. 2000;275(28):21444-21452.

13. Zeng Q, Hong W, Tan YH. Mouse PRL-2 and PRL-3, two potentially prenylated protein tyrosine phosphatases homologous to PRL-1. Biochem Biophys Res Commun. 1998;244(2):421-427.

14. Matter WF, et al. Role of PRL-3, a human muscle-specific tyrosine phosphatase, in angiotensin-II signaling. Biochem Biophys Res Commun. 2001;283(5):1061-1068.

15. Dumaual CM, Sandusky GE, Crowell PL, Randall SK. Cellular localization of PRL-1 and PRL-2 gene expression in normal adult human tissues. J Histochem Cytochem. 2006;54(12):1401-1412.

16. Lin $\mathrm{MD}$, et al. Expression of phosphatase of regenerating liver family genes during embryogenesis: an evolutionary developmental analysis among Drosophila, amphioxus, and zebrafish. BMC Dev Biol. 2013;13:18.

17. Gungabeesoon J, Tremblay ML, Uetani N. Localizing PRL-2 expression and determining the effects of dietary $\mathrm{Mg}(2+)$ on expression levels. Histochem Cell Biol. 2016;146(1):99-111.

18. Dong Y, et al. Phosphatase of regenerating liver 2 (PRL2) is essential for placental development by down-regulating PTEN (Phosphatase and Tensin Homologue Deleted on Chromosome 10) and activating Akt protein. J Biol Chem. 2012;287(38):32172-32179.

19. Dong Y, et al. Phosphatase of regenerating liver 2 (PRL2) deficiency impairs Kit signaling and spermatogenesis. J Biol Chem 2014;289(6):3799-3810.

20. Kobayashi M, et al. PRL2/PTP4A2 phosphatase is important for hematopoietic stem cell self-renewal. Stem Cells. 2014;32(7):1956-1967.

21. Cadenas S, Brand MD. Effects of magnesium and nucleotides on the proton conductance of rat skeletal-muscle mitochondria Biochem J. 2000;348 Pt 1:209-213.

22. Kozak LP, Koza RA, Anunciado-Koza R. Brown fat thermogenesis and body weight regulation in mice: relevance to humans. Int J Obes (Lond). 2010;34 Supp1 1:S23-S27.

23. Kanabrocki EL, et al. Circadian characteristics of serum calcium, magnesium and eight trace elements and of their metallo-moieties in urine of healthy middle-aged men. Clin Ter. 2008;159(5):329-346.

24. Newhouse IJ, Johnson KP, Montelpare WJ, McAuliffe JE. Variability within individuals of plasma ionic magnesium concentrations. BMC Physiol. 2002;2:6.

25. Hughes ME, et al. Harmonics of circadian gene transcription in mammals. PLoS Genet. 2009;5(4):e1000442.

26. Zhang R, Lahens NF, Ballance HI, Hughes ME, Hogenesch JB. A circadian gene expression atlas in mammals: implications for 
biology and medicine. Proc Natl Acad Sci USA. 2014;111(45):16219-16224.

27. Hoogerwerf WA, et al. Transcriptional profiling of mRNA expression in the mouse distal colon. Gastroenterology. 2008;135(6):2019-2029.

28. Pizarro A, Hayer K, Lahens NF, Hogenesch JB. CircaDB: a database of mammalian circadian gene expression profiles. Nucleic Acids Res. 2013;41(Database issue):D1009-D1013.

29. Koike N, et al. Transcriptional architecture and chromatin landscape of the core circadian clock in mammals. Science. 2012;338(6105):349-354.

30. Jothi R, Cuddapah S, Barski A, Cui K, Zhao K. Genome-wide identification of in vivo protein-DNA binding sites from ChIPSeq data. Nucleic Acids Res. 2008;36(16):5221-5231.

31. Zhang EE, et al. A genome-wide RNAi screen for modifiers of the circadian clock in human cells. Cell. 2009;139(1):199-210.

32. Bass J, Takahashi JS. Circadian integration of metabolism and energetics. Science. 2010;330(6009):1349-1354.

33. Bai Y, et al. Role of phosphatase of regenerating liver 1 (PRL1) in spermatogenesis. Sci Rep. 2016;6:34211.

34. Buchholz AC, Rafii M, Pencharz PB. Is resting metabolic rate different between men and women? Br J Nutr. 2001;86(6):641-646.

35. Ferraro R, Lillioja S, Fontvieille AM, Rising R, Bogardus C, Ravussin E. Lower sedentary metabolic rate in women compared with men. J Clin Invest. 1992;90(3):780-784.

36. Cannon B, Nedergaard J. Brown adipose tissue: function and physiological significance. Physiol Rev. 2004;84(1):277-359.

37. Valle A, García-Palmer FJ, Oliver J, Roca P. Sex differences in brown adipose tissue thermogenic features during caloric restriction. Cell Physiol Biochem. 2007;19(1-4):195-204.

38. Virtanen KA, et al. Functional brown adipose tissue in healthy adults. N Engl J Med. 2009;360(15):1518-1525.

39. van Marken Lichtenbelt WD, et al. Cold-activated brown adipose tissue in healthy men. N Engl J Med. 2009;360(15):1500-1508. 40. Cypess AM, et al. Identification and importance of brown adipose tissue in adult humans. N Engl J Med. 2009;360(15):1509-1517.

41. Pfannenberg C, et al. Impact of age on the relationships of brown adipose tissue with sex and adiposity in humans. Diabetes. 2010;59(7):1789-1793.

42. Ouellet V, et al. Outdoor temperature, age, sex, body mass index, and diabetic status determine the prevalence, mass, and glucose-uptake activity of 18F-FDG-detected BAT in humans. J Clin Endocrinol Metab. 2011;96(1):192-199.

43. Goubern M, Rayssiguier Y, Miroux B, Chapey MF, Ricquier D, Durlach J. Effect of acute magnesium deficiency on the masking and unmasking of the proton channel of the uncoupling protein in rat brown fat. Magnes Res. 1993;6(2):135-143.

44. Zurlo F, Larson K, Bogardus C, Ravussin E. Skeletal muscle metabolism is a major determinant of resting energy expenditure. J Clin Invest. 1990;86(5):1423-1427.

45. Cannon B, Nedergaard J. Nonshivering thermogenesis and its adequate measurement in metabolic studies. J Exp Biol. 2011;214(Pt 2):242-253.

46. Yamanaka R, et al. Mitochondrial $\mathrm{Mg}(2+)$ homeostasis decides cellular energy metabolism and vulnerability to stress. Sci Rep 2016;6:30027.

47. Gout E, Rébeillé F, Douce R, Bligny R. Interplay of Mg2+, ADP, and ATP in the cytosol and mitochondria: unravelling the role of Mg2+ in cell respiration. Proc Natl Acad Sci USA. 2014;111(43):E4560-E4567.

48. Wang CY, et al. Molecular cloning and characterization of a novel gene family of four ancient conserved domain proteins (ACDP). Gene. 2003;306:37-44.

49. Gómez-García I, et al. Purification, crystallization and preliminary crystallographic analysis of the CBS-domain pair of cyclin M2 (CNNM2). Acta Crystallogr Sect F Struct Biol Cryst Commun. 2012;68(Pt 10):1198-1203.

50. Hardie DG, Ross FA, Hawley SA. AMPK: a nutrient and energy sensor that maintains energy homeostasis. Nat Rev Mol Cell Biol. 2012;13(4):251-262.

51. Hirata Y, Funato Y, Takano Y, Miki H. Mg2+-dependent interactions of ATP with the cystathionine- $\beta$-synthase (CBS) domains of a magnesium transporter. J Biol Chem. 2014;289(21):14731-14739.

52. Corral-Rodríguez MÁ, et al. Nucleotide binding triggers a conformational change of the CBS module of the magnesium transporter CNNM2 from a twisted towards a flat structure. Biochem J. 2014;464(1):23-34.

53. Giménez-Mascarell P, et al. Structural Basis of the Oncogenic Interaction of Phosphatase PRL-1 with the Magnesium Transporter CNNM2. J Biol Chem. 2017;292(3):786-801.

54. Panda S, et al. Coordinated transcription of key pathways in the mouse by the circadian clock. Cell. 2002;109(3):307-320.

55. Reszka E, Przybek M. Circadian Genes in Breast Cancer. Adv Clin Chem. 2016;75:53-70.

56. Blakeman V, Williams JL, Meng QJ, Streuli CH. Circadian clocks and breast cancer. Breast Cancer Res. 2016;18(1):89.

57. Schneider CA, Rasband WS, Eliceiri KW. NIH Image to ImageJ: 25 years of image analysis. Nat Methods. 2012;9(7):671-675.

58. de Baaij JH, et al. Membrane topology and intracellular processing of cyclin M2 (CNNM2). J Biol Chem. 2012;287(17):13644-13655.

59. Hardy S, Wong NN, Muller WJ, Park M, Tremblay ML. Overexpression of the protein tyrosine phosphatase PRL-2 correlates with breast tumor formation and progression. Cancer Res. 2010;70(21):8959-8967.

60. Klimcakova E, et al. PGC-1 $\alpha$ promotes the growth of ErbB2/Neu-induced mammary tumors by regulating nutrient supply. Cancer Res. 2012;72(6):1538-1546.

61. Morita M, et al. mTORC1 controls mitochondrial activity and biogenesis through 4E-BP-dependent translational regulation Cell Metab. 2013;18(5):698-711.

62. Tschöp MH, et al. A guide to analysis of mouse energy metabolism. Nat Methods. 2011;9(1):57-63.

63. Wu C, et al. BioGPS: an extensible and customizable portal for querying and organizing gene annotation resources. Genome Biol. 2009;10(11):R130.

64. Bugge A, et al. Rev-erb $\alpha$ and Rev-erb $\beta$ coordinately protect the circadian clock and normal metabolic function. Genes Dev. $2012 ; 26(7): 657-667$ 